\title{
Energies and widths of atomic core-levels in liquid mercury
}

\author{
Y.-P. Maillard ${ }^{\mathrm{a}}$, J.-Cl. Dousse, and J. Hoszowska \\ Department of Physics, University of Fribourg, Chemin du Musée 3, 1700 Fribourg, Switzerland
}

Received 2 September 2009 / Received in final form 22 December 2009

Published online 16 February 2010 - (C) EDP Sciences, Società Italiana di Fisica, Springer-Verlag 2010

\begin{abstract}
High-resolution measurements of the photoinduced X-ray emission of liquid mercury were performed, using a transmission DuMond-type crystal spectrometer for transitions above $11 \mathrm{keV}$ and a reflection von Hamos-type crystal spectrometer for transitions below $11 \mathrm{keV}$. The target X-ray fluorescence was produced by irradiating the sample with the Bremsstrahlung from X-ray tubes. The energies and natural linewidths of $6 \mathrm{~K}$-shell, $26 \mathrm{~L}$-shell and $2 M$-shell X-ray transitions were determined. Using a least-squaresfit method to solve the two sets of equations derived from the observed transition energies and transition widths the binding energies of the subshells $K$ to $M_{5}$ and $O_{1}$ and the level widths of the subshells $K$ to $N_{5}$ and $O_{1}$ could also be determined.
\end{abstract}

\section{Introduction}

Mercury is the only common metal liquid at room temperature. It has been widely used in the past for making thermometers, barometers, diffusion pumps, vapor lamps, advertising signs, and electrical switches. Pesticides, dental preparations, batteries and catalysts are further examples of the use of the versatile liquid metal in everyday life. However, mercury is also known as a virulent poison. Furthermore, since $\mathrm{Hg}$ is a very volatile element, dangerous levels are easily attained in air. The danger is still increased by the colorless and odorless nature of the $\mathrm{Hg}$ vapor. Because mercury wastes have not been always eliminated properly in the past decades, this dangerous metal has spread out and accumulated in nature. As a consequence the toxic element has received a renewed interest in the recent years and mercury pollution has become an important field in environmental studies.

Among the methods employed to detect traces of mercury, the X-ray fluorescence (XRF) and particle-induced $\mathrm{X}$-ray emission (PIXE) techniques are probably the most efficient ones. In these techniques, the concentrations of the elements of interest are usually determined from the relative yields of the sample $K \mathrm{X}$-ray lines. Energies of the latter have thus to be known accurately to identify unambiguously the observed transitions. In in-situ XRF analysis of $\mathrm{Hg}$-contaminated samples, portable X-ray sources characterized by high-voltages limited to $40-60 \mathrm{kV}$ are employed that restrict the observation to the $L$ X-ray emission of mercury. In this case, a correct and reliable analysis of the complex $L$ X-ray spectrum in which many transitions overlap is only possible if the energies of the $\mathrm{Hg} L$ $\mathrm{X}$-ray transitions are known with an accuracy of the order of $1 \mathrm{eV}$. In the XRF and PIXE methods, the knowledge of the line shapes of the measured X-ray transitions rep-

\footnotetext{
a e-mail: yves-patrik.maillard@unifr.ch
}

resents an additional important asset to improve the precision and reliability of the results even in low-resolution measurements [1].

On the other hand, $\mathrm{Hg}$ is the heaviest stable element having all electronic subshells filled in the ground state. Precise experimental data concerning the transition energies and transition widths of mercury are therefore particularly adequate to probe the goodness of atomic structure calculations of heavy elements. However, as indicated by Deslattes et al. [2], for mercury the only existing data base for experimental X-ray transition energies is that of Bearden [3] which is more than 40 years old. Furthermore, as it was pointed out by Fuggle already in 1980 [4], a similar lack of experimental information does exist for the widths of the atomic core-levels of this element. The situation has not much improved in the last two decades. In the recent inventory of atomic level widths made by Campbell and Papp [5] one can see indeed that for mercury experimental data are available only for the $L_{1-3^{-}}$ subshells [6] and the $N_{4-7}$-subshells $[7,8]$. Here also there is thus a clear need for modern data for the widths of most $\mathrm{Hg}$ core-levels.

For the above-mentioned reasons we have performed a series of high-resolution X-ray emission spectroscopy (XES) measurements of liquid $\mathrm{Hg}$. The transition energies and transition widths of a variety of $K, L$ and $M$ X-ray lines induced by means of photoionization were determined. As the energy, respectively the width, of an X-ray transition is given by the difference of the binding energies of the two atomic levels involved in that transition, respectively by the sum of the widths of the two levels, two sets of linear equations could be derived from the measured transition energies and transition widths. Solving the two systems of equations by means of a least-squares-fit method, two self-consistent sets of experimental electron binding energies and level widths could be obtained for most corelevels of liquid $\mathrm{Hg}$. 

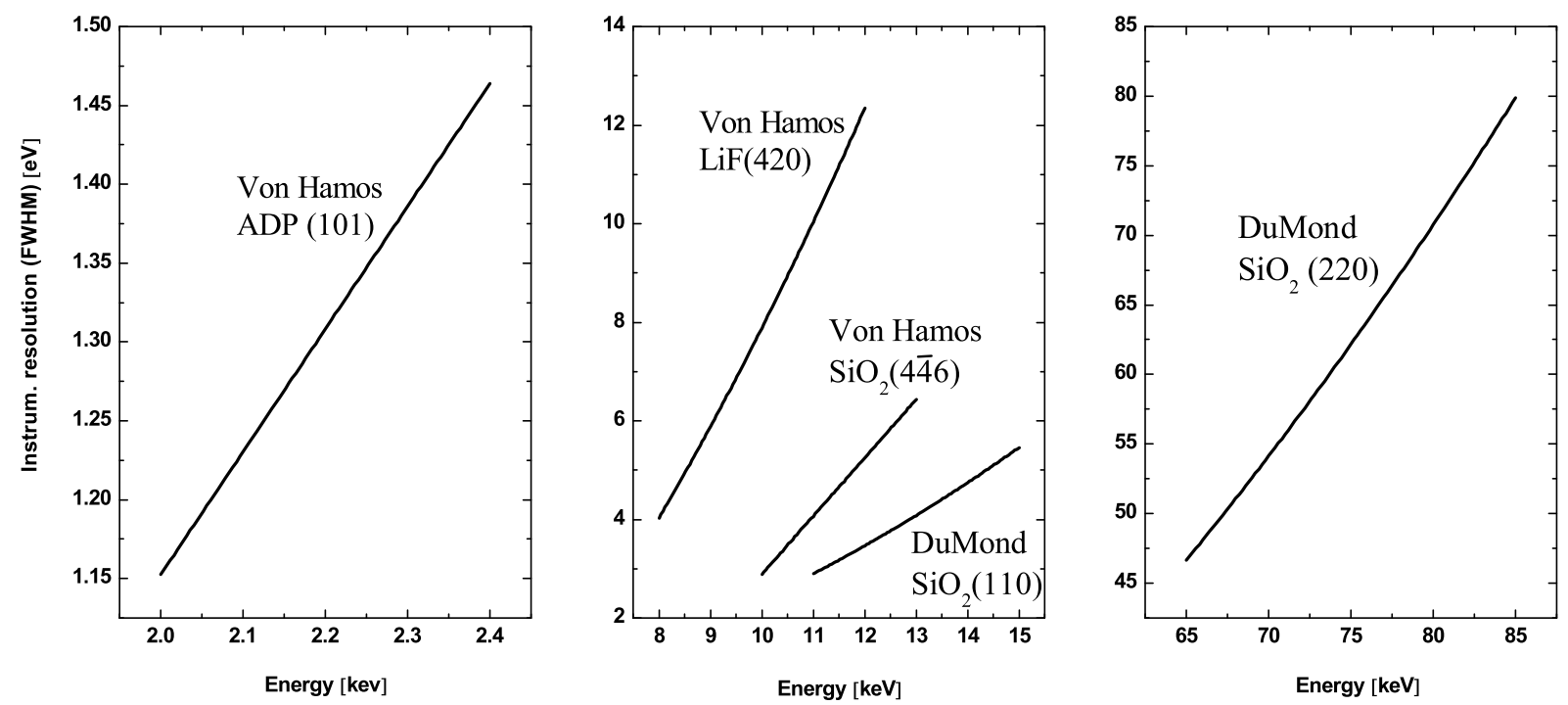

Fig. 1. Variation of the FWHM instrumental resolution of the von Hamos and DuMond spectrometers as a function of the X-ray energy. The energy-dependent instrumental broadening is shown for the energy domains corresponding to the measured $M$ transitions (left panel), $L$ transitions (mid panel) and $K$ transitions (right panel).

\section{Experiment}

\subsection{Experimental method}

The measurements were performed at the University of Fribourg by means of high-resolution X-ray spectroscopy. The $K$ and most of the $L \mathrm{X}$-ray lines were observed with a DuMond transmission-type bent crystal spectrometer. As in the transmission geometry (Laue diffraction) the photon absorption in the crystal becomes very important at low energies, the $L$ transitions below $11 \mathrm{keV}$ and the $M$ transitions were measured with a von Hamos reflectiontype bent crystal spectrometer (Bragg diffraction). The energy domains covered by the two spectrometers overlapping between 11 and $15 \mathrm{keV}$, the $L_{2}-M_{4}$ transition $(E=11.8 \mathrm{keV})$ was measured with both instruments in order to cross-check the obtained results and to probe the reliability of the quoted uncertainties.

The target consisted of $99.999 \%$ pure liquid mercury enclosed in a stainless steel cell sealed with a $75 \mu \mathrm{m}$-thick Kapton $^{\mathrm{TM}}$ foil. For the $M \mathrm{X}$-ray measurements a thinner foil $(8 \mu \mathrm{m})$ was used in order to diminish the absorption of the $2.2 \mathrm{keV}$ fluorescence X-rays in the target window. For the production of the mercury fluorescence, the liquid target was irradiated with the Bremsstrahlung from commercial 3-kW Coolidge-type X-ray tubes. The choice of the X-ray tube ( $\mathrm{Au}$ or $\mathrm{Cr}$ anode) and the values of the high-voltage and current were optimized for each series of measurements in order to obtain the best peak-tobackground ratios.

\subsection{DuMond spectrometer}

The DuMond crystal spectrometer of Fribourg consists mainly of a target chamber, a cylindrically bent crystal plate that can be rotated around a vertical axis by $\pm 20^{\circ}$ and a self-propelled detector-collimator system. For the present project the spectrometer was operated in the socalled slit-geometry. In this geometry the sample is viewed by the crystal through a narrow rectangular slit located on the Rowland circle, a few $\mathrm{cm}$ in front of the target. For X-ray measurements, this geometry presents several advantages with respect to the standard DuMond geometry. In particular the slit-geometry makes possible the use of extended radiation sources. This is an important asset when measuring gaseous $[9,10]$ or liquid samples. Furthermore, it permits one to get rid of the systematic errors originating from the thermal deformation of the irradiated sample and to enhance the luminosity of the spectrometer by diminishing the self-absorption of the fluorescence $\mathrm{X}$-rays in the target. The $30-\mathrm{mm}$ high rectangular slit was made of two vertical 5-mm thick juxtaposed $\mathrm{Pb}$ plates. For the present measurements a slit width of $0.15 \mathrm{~mm}$ was employed. More technical details about the DuMond spectrometer can be found in [11].

In the DuMond geometry the crystal plate is bent cylindrically and the radius of curvature is twice that of the focal circle. For this project a $10 \times 10 \mathrm{~cm}^{2}(110)$ quartz crystal plate with a thickness of $0.5 \mathrm{~mm}$ was employed. The plate was bent to a radius of $311 \mathrm{~cm}$ by means of a clamping block similar to the one described in [12]. Due to the window width of the clamping block and because the top and bottom parts of the crystal which are poorly curved as a result of the Sumbaev effect [13] could not be used, the effective diffracting area of the crystal was $12 \mathrm{~cm}^{2}$. The corresponding solid angle of the spectrometer was thus about $1.2 \times 10^{-4} \mathrm{sr}$. The Bragg angles were measured by means of a Doppler effect-based laser interferometer with a precision of a few milli-arcsec [14].

For the detection of the diffracted X-rays, a 5-in.diameter Phoswich scintillation counter was employed. The latter consists of a thin (0.25 in.) NaI(Tl) crystal 
followed by an optically coupled thicker (2 in.) $\mathrm{Cs}(\mathrm{Tl})$ crystal. Both crystals are mounted on the same photomultiplier tube. As the rising times of the signals are different for the two crystals, the events corresponding to each scintillation can be identified by a pulse-shape analysis [15]. This type of detector permits to strongly diminish the background arising from high-energy photons, which is an important asset when weak X-ray lines have to be extracted from the background [16-18]. A further reduction of the background was obtained by enclosing the Phoswich detector in a heavy $\mathrm{Pb}-\mathrm{Cu}-\mathrm{Al}$ shielding, and by sorting on-line the events of interest as a function of their energy.

The angular instrumental response of the DuMond spectrometer depends principally on the slit width and on the precision of the crystal curvature but not, at least in first approximation, on the Bragg angle. In general, the response can be well reproduced by a Gaussian function. The angular instrumental resolution was determined by measuring the $K \alpha_{1}$ transitions of $\mathrm{Gd}$ and $\mathrm{Au}$, in first and fourth orders of diffraction, respectively, using in the fits the level widths quoted by Campbell and Papp [5] to derive the Lorentzian widths of the two transitions. From the fits in which the Lorentzian widths were kept fixed, consistent FWHM Gaussian widths of 12.8(7) arcsec (Gd) and $12.9(1.6) \operatorname{arcsec}(\mathrm{Au})$ were found.

The energy resolution $\Gamma_{E}$ can be deduced from the angular resolution $\Gamma_{\theta}$ using the following relation:

$$
\Gamma_{E}=\cot (\theta) E \Gamma_{\theta}
$$

where $\theta$ is the Bragg angle and $E$ represents the photon energy. As shown by (1), for a given photon energy, $\Gamma_{E}$ improves with increasing Bragg angles. For this reason the $K \mathrm{X}$-rays were measured in second order of diffraction. The variation of the energy resolution with the photon energy is depicted in Figure 1. One sees that for the $L \mathrm{X}$-ray measurements the resolution of the DuMond spectrometer varied between $3 \mathrm{eV}$ at $11 \mathrm{keV}$ and $5.5 \mathrm{eV}$ at $15 \mathrm{keV}$, whereas for the $K \mathrm{X}$-ray measurements the resolution was markedly worse, ranging from $46 \mathrm{eV}$ at $65 \mathrm{keV}$ to $80 \mathrm{eV}$ at $85 \mathrm{keV}$.

In the DuMond geometry the crystal-to-slit distance (focal length $f$ ) corresponding to the best instrumental resolution varies with the photon energy, i.e., the Bragg angle $\theta$ :

$$
f=R \cos \theta
$$

where $R$ is the radius of curvature of the crystal. As a consequence the focal length $f$ should be adjusted for each $\mathrm{X}$-ray line. However, when the X-ray spectrum extends over a large angular range and comprises many weak lines, this optimization is not so convenient. It is indeed simpler to measure a selected group of lines with an average value of the focal length and to correct then off-line the instrumental broadenings for the tiny deviations resulting from the focal length errors [11]. For this reason the variation of the instrumental broadening as a function of the focal length was determined by measuring the $K \alpha_{1}$ line of $\mathrm{Gd}$ at several different focusing distances around the optimum value.
To calibrate in energy the angular spectra, the crystal lattice spacing constant and the zero of the Bragg angle scale must be known accurately. The lattice constant of the $\mathrm{SiO}_{2}(110)$ crystal was determined by measuring the $K \alpha_{1}$ transition of $\mathrm{Au}$ in fourth order on both sides of diffraction. Using the so-determined double Bragg angle $2 \theta$ and the wavelength of the $\mathrm{Au} K \alpha_{1}$ transition reported in $[19,20]$, the value $d_{110}=2.456645(20) \AA$ was obtained. As the $K \mathrm{X}$-ray measurements were performed about two years later the lattice constant was reexamined at the end of these measurements, using again the $K \alpha_{1}$ transition of $\mathrm{Au}$ but this time in second order of diffraction. A somewhat bigger value of 2.456705(19) $\AA$ was obtained. As no explanation was found for this small but significant discrepancy, we opted to use the average value of the two measurements and for the uncertainty the half of the difference between the two values, i.e., $d_{110}=2.456675(30) \AA$. Finally, as the zero Bragg angle may vary with the focal length $f$, it was determined for each group of lines observed at the same value of $f$ by measuring the most intense transition of the group at positive and negative Bragg angles.

With the DuMond spectrometer, data collection is carried out point by point. In other words, the angular spectrum is scanned step by step with the same acquisition time for each angular position of the crystal. If the measured X-ray spectrum comprises weak lines, acquisition times up to thousand seconds per point or even more are needed so that the measurement may last several days. In order to minimize the systematic errors related to longterm instabilities of the experimental setup (e.g. fluctuations of the X-ray tube intensity) such angular regions were measured in several successive step-by-step scans that were then summed off-line. For illustration, a partial $L$ X-ray spectrum measured with the DuMond spectrometer is shown in Figure 2.

\subsection{Von Hamos spectrometer}

The $M_{4}-N_{6}, M_{5}-N_{7}, L_{3}-M_{5}, L_{3}-M_{4}, L_{3}-M_{1}, L_{2}-M_{1}$ and $L_{2}-M_{4}$ transitions were measured with a von Hamos Bragg-type curved crystal spectrometer. The latter consists mainly of three components: a target-slit system, a cylindrically bent crystal and a position-sensitive detector. A detailed description of the spectrometer design and its operational characteristics can be found in [21].

For the present project a slit width of $0.25 \mathrm{~mm}$ was employed. As the Bragg angles covered by the von Hamos spectrometer range between $24^{\circ}$ and $61^{\circ}$, three different crystals were needed. The $L_{3} \mathrm{X}$-ray lines were measured with a $\mathrm{LiF}(420)$ crystal $(2 d=1.8010 \AA)$, the $M$-rays with an $\operatorname{ADP}(101)$ crystal $(2 d=10.642 \AA)$, whereas the $L_{2}-M_{4}$ transition was observed in second order, using a $\mathrm{SiO}_{2}(2 \overline{2} 3)$ crystal $(2 d=2.7500 \AA)$. The three crystal plates were $10-\mathrm{cm}$ high and 5 -cm wide with thicknesses of $1.1 \mathrm{~mm}(\mathrm{LiF}), 0.3 \mathrm{~mm}$ (ADP) and $0.4 \mathrm{~mm}\left(\mathrm{SiO}_{2}\right)$. They were cylindrically bent to a radius of $25.4 \mathrm{~cm}$.

The diffracted X-rays were detected with a deepdepleted charge-coupled-device (CCD) camera (depletion 


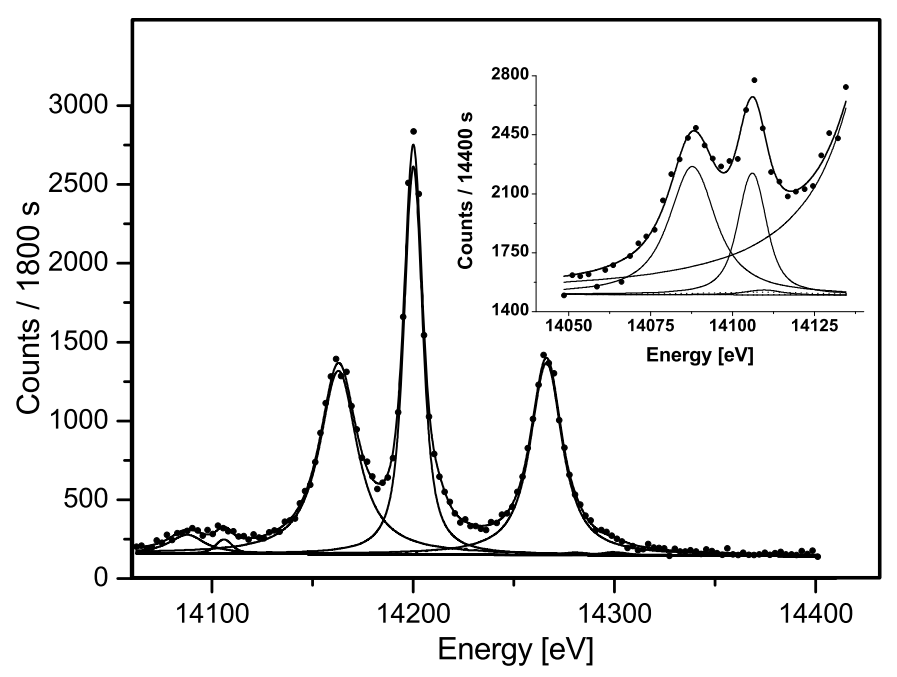

Fig. 2. Part of the $\mathrm{Hg} L \mathrm{X}$-ray spectrum measured with the DuMond spectrometer. The spectrum was corrected for the $L_{2}$ absorption edge occurring at $14209 \mathrm{eV}$. The three main lines correspond to the $L_{1}-N_{2}$ (at $\left.14160 \mathrm{eV}\right), L_{2}-O_{4}$ and $L_{1}-N_{3}$ transitions. The low energy part of the spectrum around $14100 \mathrm{eV}$ was remeasured with a longer acquisition time. It is shown enlarged in the inset where one can distinguish the $L_{2}-O_{1}$ (at $14088 \mathrm{eV}$ ) and $L_{2}-N_{6}$ transitions as well as the weak $L_{2}-N_{7}$ transition (solid line at $14110 \mathrm{eV}$ ) overlapping with the $L_{2}-O_{1} N$-satellite structure (dotted line). The dotted line at about $14220 \mathrm{eV}$ (in the main figure) corresponds to the $\mathrm{N}$-satellite of the $\mathrm{L}_{2}-\mathrm{O}_{4}$ transition. The hardly visible components around $14280 \mathrm{eV}$ and $14300 \mathrm{eV}$, respectively, correspond to the $L_{1}-O_{2}$ and $L_{1}-O_{3}$ transitions of Au. The latter are due to the elastic scattering by the target of the characteristic X-ray emission from the X-ray tube anode.

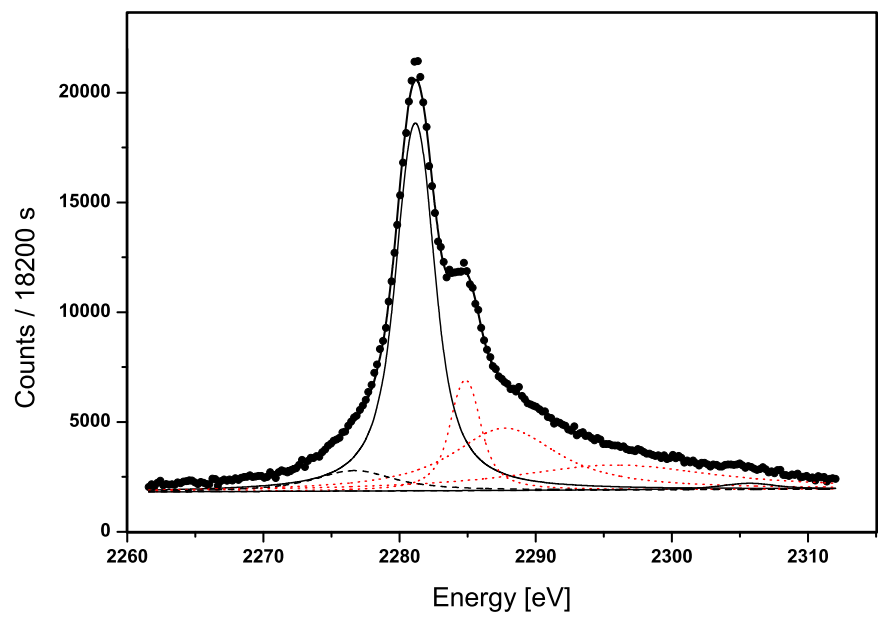

Fig. 3. (Color online) $\mathrm{Hg} M_{4}-N_{6}$ transition measured with the von Hamos spectrometer. The complex $N$-satellite structure was fitted with three Voigtians (dotted lines). The dashed line around $2275 \mathrm{eV}$ accounts for the slight asymmetry observed in the instrumental response of the von Hamos spectrometer when equipped with the ADP (101) crystal. The solid line at about $2305 \mathrm{eV}$ was assigned to residuals from the sixth order diffraction of the $L_{2}-M_{4}$ transition. depth of $50 \mu \mathrm{m}$ ) consisting of 1024 pixels in the direction of dispersion and 256 pixels in the vertical direction with a pixel size of $27 \times 27 \mu \mathrm{m}^{2}$ [22]. The CCD chip was thermoelectrically cooled down to $-60{ }^{\circ} \mathrm{C}$. For illustration the X-ray spectrum corresponding to the $M_{4}-N_{6}$ transition is depicted in Figure 3.

The energy calibration of the X-ray spectra was based on photoionization measurements of the $K \alpha_{1}$ and $L \alpha_{1}$ transitions of several mid-heavy elements. For the calibration of the spectra measured with the $\operatorname{LiF}(420)$ and $\mathrm{SiO}_{2}(2 \overline{2} 3)$ crystals, the $K \alpha_{1}$ transitions of $\mathrm{Zn}, \mathrm{Ge}$, Se and $\mathrm{Br}$ were employed, whereas for the calibration of the spectra measured with the $\operatorname{ADP}(101)$ crystal the $L \alpha_{1}$ transitions of $\mathrm{Nb}$ and Mo were used. The energies of the six calibration transitions are given as footnotes in Table 1 (see Sect. 4). They were derived from the wavelengths reported by Bearden [3]. Since the latter values are given in the $\AA^{*}$ scale, they were corrected by the conversion factor $1.0000150 \AA^{*} / \AA$ and then converted to energies using the energy-wavelength product $V \lambda=12.398419 \mathrm{keV} \AA$. The two conversion factors were computed using for the Planck constant $h$, speed of light $c$ and elementary charge $e$ the values recommended in 2006 by the Committee on Data for Science and Technology (CODATA) [23]. Furthermore, the uncertainties quoted by Bearden being probable errors (50\% confidence limits), they were multiplied by 1.48 to obtain standard deviation errors (67\% confidence limits).

The instrumental broadening of the von Hamos spectrometer originates mainly from the slit width, the crystal mosaicity width and the spatial resolution of the detector. The broadening due to the slit is given by:

$$
\Delta E_{\text {slit }}=\frac{w_{\text {slit }}}{2 R} \cos (\theta) E
$$

where $w_{\text {slit }}$ represents the width of the slit, $R$ the crystal radius of curvature, $\theta$ the Bragg angle and $E$ the energy of the measured transition. Similarly the broadening resulting from the spatial resolution of the detector can be written as:

$$
\Delta E_{C C D}=\frac{\Delta x}{4 R} \sin (2 \theta) E
$$

where $\Delta x$ stands for the size of the CCD pixels. From the above relations, one finds that for the $M$ and $L$ X-ray lines measured with the von Hamos spectrometer the broadening related to the slit ranged between $0.9 \mathrm{eV}$ and $4.0 \mathrm{eV}$, whereas the detector contribution varied between $0.05 \mathrm{eV}$ and $0.31 \mathrm{eV}$, only. The $27 \mu \mathrm{m}$ resolution of the CCD detector was thus not really needed and a software binning of four adjacent columns was performed off-line in order to obtain higher counting rates in the one-dimensional energy spectra.

The instrumental broadening was determined experimentally using the above-mentioned $K \alpha_{1}$ and $L \alpha_{1}$ calibration measurements. It was found that for the three crystals the instrumental response could be well reproduced by a Gaussian function. The full widths at half maximum (FWHM) of the three Gaussians were obtained from the fits of the calibration measurements in which the natural Lorentzian widths of the transitions were kept 
Table 1. Energies in eV of the observed $\mathrm{Hg}$ transitions. The energies quoted in the third column were derived from the $K$ to $M_{5}$ and $O_{1}$ binding energies obtained in the present work by the least-squares fit method (see Sect. 4.2) and the average values of the $N_{1}$ to $N_{7}$ and $O_{2}$ to $O_{5}$ binding energies reported by Svensson et al. [7] and Fuggle and Martensson [4]. The notation $68892.78(1.10 / 29)$ means $68892.78 \mathrm{eV} \pm 1.10 \mathrm{eV}$ with an included statistical error from the fit of $\pm 0.29 \mathrm{eV}$.

\begin{tabular}{|c|c|c|c|c|}
\hline Transition & Present work & $\begin{array}{c}\text { Calculated from present } \\
\text { binding energies }\end{array}$ & Bearden $[3]^{a}$ & $\begin{array}{l}\text { Theoretical values } \\
\text { from }[2]\end{array}$ \\
\hline$K-L_{2}$ & $68892.78(1.10 / 29)^{b}$ & $68893.14(48)$ & $68895.1(1.7)$ & $68894.3(2.3)$ \\
\hline$K-L_{3}$ & $70818.53(89 / 21)^{b}$ & $70818.15(47)$ & $70819.5(1.8)$ & $70819.0(2.2)$ \\
\hline$K-M_{2}$ & $79822.94(1.16 / 63)^{b}$ & $79822.65(50)$ & $79823.3(2.3)$ & $79824.1(3.6)$ \\
\hline$K-M_{3}$ & $80253.86(1.04 / 35)^{b}$ & $80254.22(50)$ & $80254.2(2.3)$ & $80256.1(3.7)$ \\
\hline$K-N_{3}$ & $82525.88(1.25 / 50)^{b}$ & $82526.00(49)$ & $82544.8(16.3)$ & $82527.7(2.5)$ \\
\hline$L_{1}-M_{2}$ & $11563.09(18 / 8)^{c}$ & $11563.10(17)$ & $11563.1(1.1)$ & $11563.7(3.0)$ \\
\hline$L_{1}-M_{3}$ & $11994.68(20 / 8)^{c}$ & 11994.67(19) & $11995.4(1.2)$ & 11995.6(3.1) \\
\hline$L_{1}-M_{4}$ & $12457.10(54 / 51)^{c}$ & $12456.96(17)$ & $12445.5(3.7)$ & $12456.6(2.4)$ \\
\hline$L_{1}-M_{5}$ & $12547.68(31 / 26)^{c}$ & $12547.65(17)$ & $12560.3(3.8)$ & $12547.1(2.3)$ \\
\hline$L_{1}-N_{2}$ & $14162.74(27 / 18)^{c}$ & $14162.59(43)$ & $14162.3(1.7)$ & $14164.7(4.6)$ \\
\hline$L_{1}-N_{3}$ & $14266.05(26 / 16)^{c}$ & $14266.44(22)$ & $14264.8(1.7)$ & $14267.2(1.9)$ \\
\hline$L_{1}-N_{5}$ & $14484.14(47 / 42)^{c}$ & $14484.14(29)$ & $14474.3(1.8)$ & $14484.8(1.7)$ \\
\hline$L_{1}-O_{3}$ & $14778.86(28 / 18)^{c}$ & $14778.44(29)$ & $14778.5(1.8)$ & \\
\hline$L_{2}-M_{1}$ & $10648.51(71 / 12)^{d}$ & $10647.06(32)$ & 10651.4(1.4) & $10647.3(2.5)$ \\
\hline \multirow[t]{2}{*}{$L_{2}-M_{4}$} & $11823.29(15 / 2)^{c}$ & $11823.38(11)$ & $11822.7(8)$ & $11822.7(1.4)$ \\
\hline & $11823.75(95 / 4)^{e}$ & & & \\
\hline$L_{2}-N_{1}$ & $13407.08(29 / 22)^{c}$ & $13407.01(41)$ & $13410.3(1.5)$ & 13408.1(3.4) \\
\hline$L_{2}-N_{4}$ & $13831.30(20 / 5)^{c}$ & $13831.26(18)$ & $13830.2(1.1)$ & $13831.7(2.7)$ \\
\hline$L_{2}-N_{6}$ & $14105.81(30 / 23)^{c}$ & $14105.61(15)$ & 14107.3(1.7) & $14105.2(1.6)$ \\
\hline$L_{2}-O_{1}$ & $14087.57(42 / 37)^{c}$ & $14087.79(27)$ & 14089.7(1.7) & \\
\hline$L_{2}-O_{4}$ & $14199.86(22 / 9)^{c}$ & $14199.91(15)$ & $14198.8(1.7)$ & \\
\hline$L_{3}-M_{1}$ & $8721.71(34 / 3)^{d}$ & $8722.04(30)$ & $8721.3(9)$ & $8722.6(1.9)$ \\
\hline$L_{3}-M_{4}$ & $9898.71(32 / 6)^{d}$ & $9898.36(13)$ & $9897.7(8)$ & 9898.1(1.3) \\
\hline$L_{3}-M_{5}$ & $9989.43(33 / 1)^{d}$ & $9989.05(14)$ & $9988.9(6)$ & $9988.6(1.2)$ \\
\hline$L_{3}-N_{1}$ & $11482.01(23 / 17)^{c}$ & $11482.00(41)$ & $11482.5(1.1)$ & $11483.5(3.2)$ \\
\hline$L_{3}-N_{4}$ & $11905.64(22 / 12)^{c}$ & $11906.25(18)$ & 11904.1(1.2) & $11907.0(2.5)$ \\
\hline$L_{3}-N_{5}$ & $11925.58(18 / 2)^{c}$ & $11925.55(27)$ & $11924.2(1.2)$ & $11926.3(6)$ \\
\hline$L_{3}-N_{7}$ & $12184.32(42 / 38)^{c}$ & $12184.70(15)$ & $12194.1(1.2)$ & $12184.7(1.4)$ \\
\hline$L_{3}-O_{1}$ & $12162.92(33 / 28)^{c}$ & $12162.78(24)$ & $12162.6(1.2)$ & \\
\hline$L_{3}-O_{5}$ & $12277.00(17 / 4)^{c}$ & $12276.80(15)$ & $12277.1(1.3)$ & \\
\hline$M_{4}-N_{6}$ & $2282.23(5 / 1)^{f}$ & $2282.23(13)$ & $2282.5(6)$ & \\
\hline$M_{5}-N_{7}$ & $2195.71(9 / 1)^{f}$ & $2195.65(15)$ & $2195.3(5)$ & \\
\hline
\end{tabular}

${ }^{a}$ Readjusted according to CODATA 06 [23].

${ }^{b}$ DuMond spectrometer, $\mathrm{SiO}_{2}$ (220). Reference energy: 68804.50(18) eV (Au K $\left.\alpha_{1}\right)$ [2].

${ }^{c}$ DuMond spectrometer, $\mathrm{SiO}_{2}$ (110). Reference energy: 68804.50(18) eV (Au Ko $\left.\alpha_{1}\right)$ [2].

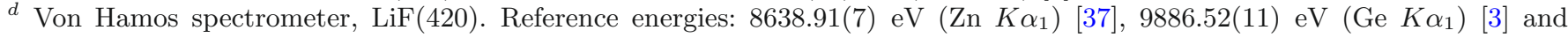
$11222.52(12) \mathrm{eV}\left(\mathrm{Se} K \alpha_{1}\right)[2]$.

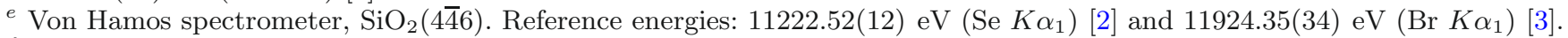

${ }^{f}$ Von Hamos spectrometer, $\operatorname{ADP}(101)$. Reference energies: 2165.90(11) eV (Nb $\left.L \alpha_{1}\right)$ [3] and 2293.18(5) eV (Mo $\left.L \alpha_{1}\right)$ [3].

fixed at the values derived from the atomic level widths quoted in the tables of Campbell and Papp [5]. The variation of the instrumental resolution with the photon energy is shown for the three crystals in Figure 1. One can see that for the energy corresponding to the $L_{2}-M_{4}$ transition $(11.8 \mathrm{keV})$ the resolution obtained with the $\mathrm{SiO}_{2}(4 \overline{4} 6)$ crystal $(5 \mathrm{eV})$ was markedly better than the one obtained with the $\mathrm{LiF}(420)$ crystal $(15 \mathrm{eV})$. Since the slit and detector contributions to the broadening are about equal for the two crystals, the observed resolution difference indicates that the mosaicity of the LiF crystal is more pronounced. From Figure 1 one can also see that in the $11-15 \mathrm{keV}$ overlapping energy region the resolution of the von Hamos spectrometer, even when using the $\mathrm{SiO}_{2}(4 \overline{4} 6)$ crystal, is somewhat worse than the one of the DuMond spectrometer, justifying thus the choice of the transmission-type instrument for the measurement of the $L$ X-ray transitions above $11 \mathrm{keV}$. 


\section{Data analysis}

\subsection{Fitting procedure}

The transitions observed with both spectrometers were analysed by means of the least-squares-fitting software package MINUIT [24]. Since the widths of the Gaussian instrumental responses of the two spectrometers were comparable to the widths of the Lorentzian functions corresponding to the line shapes of the X-ray transitions, Voigt profiles were employed to reproduce the line shapes of the measured transitions [25]. The energies, intensities and Lorentzian widths of the transitions as well as the two parameters of the linear background were let free in the fitting procedure, whereas the Gaussian widths were kept fixed at their known values. However, in several cases, some additional constraints needed to be set. For instance, in the analysis of the $K \beta_{2}$ spectrum, the intensity ratio of the $K-N_{2}$ and $K-N_{3}$ transitions and the energy separation between the two components had to be kept fixed. The intensity ratio was deduced from the tables of Scofield [26] and the energy difference from the average values of the $N_{2}$ and $N_{3}$ subshell binding energies quoted in $[4,7]$. Similar constraints were applied to the fits of the $L_{1}-N_{4,5}, L_{1}-O_{2,3}, L_{3}-N_{6,7}$ and $L_{3}-O_{4,5}$ doublets.

Typical examples of the difficulties encountered during the data analysis are illustrated in the spectrum shown in Figure 2. The three lines that clearly emerge from the background correspond to the $L_{1}-N_{2}$ (at about $14160 \mathrm{eV}), L_{2}-O_{4}$ and $L_{1}-N_{3}$ transitions. The weaker structure around $14100 \mathrm{eV}$ was assigned to the $L_{2}-O_{1}$ and $L_{2}-N_{6}$ transitions. The asymmetry occurring on the high-energy flank of the $L_{2}-O_{4}$ transition is due to unresolved $N$-satellite structures originating from $L_{1} L_{2} N$ Coster-Kronig transitions. Finally, the excess intensity around $14300 \mathrm{eV}$ corresponds to the elastic scattering by the $\mathrm{Hg}$ target of the characteristic $L_{1}-O_{2}$ and $L_{1}-O_{3}$ transitions of the $\mathrm{Au} \mathrm{X}$-ray tube anode. In addition to the problems related to the large number of transitions appearing in that energy region and the overlap of some of these transitions, a further difficulty concerned the presence in the spectrum of the $L_{2}$ edge (at $14209 \mathrm{eV}$ ). The line shapes of the $L_{2}-O_{4}$ and $L_{1}-N_{3}$ were indeed found to be affected by this edge as a result of the abrupt change of the self-absorption of the photons of interest in the target. Thus the spectrum was first corrected using a theoretically constructed profile to reproduce the shape of the $L_{2}$ edge. The edge jump height was adjusted so that the relative yields of the $L_{1}-N_{2}$ and $L_{1}-N_{3}$ transitions that lie respectively below and above the $L_{2}$ edge were consistent with the relative intensities reported by Scofield [26]. The corrected spectrum was then fitted, using one Voigtian for each of the seven transitions. No constraint was set except for the $\mathrm{L}_{2}-\mathrm{O}_{4} \mathrm{~N}$-satellite whose energy and width were fixed at values derived from the fit of the $L_{3}-O_{5}$ $N$-satellite which could be resolved. The errors of the energies and widths of the $L_{2}-O_{4}$ and $L_{1}-N_{3}$ transitions induced by this crude approximation were found to be small because of the weak relative intensity of the $L_{2}-O_{4}$ $N$-satellite (about 6\%).
To improve the poor statistics observed for the $L_{2}-O_{1}$ and $L_{2}-N_{6}$ transitions, the energy region between 14050 and $14135 \mathrm{eV}$ was remeasured in several successive scans corresponding to a total collecting time of $4 \mathrm{~h}$ per point (see inset of Fig. 2). This region was fitted separately with four Voigtians, two for the diagram transitions plus one for the $L_{2}-N_{7}$ transition (thin solid line in the inset, at $14110 \mathrm{eV}$ ) and one for the $L_{2}-O_{1} N$-satellite (dotted line). As shown in the inset, the four lines are superimposed on the low-energy tail of the stronger $L_{1}-N_{2}$ transition whose position and width were kept fixed, using the values obtained from the fit of the full spectrum. The intensity, however, was let free to adjust the intensity scale of the two spectra.

The spectra measured with the von Hamos spectrometer were analyzed in a similar way. An example is presented in Figure 3 showing the $M_{4}-N_{6}$ transition. The latter could be well fitted with a single Voigt profile. The small component fitted below the main line is due to the ADP (101) crystal. The same asymmetry was indeed observed in the $L \alpha_{1}$ transitions of $\mathrm{Nb}$ and Mo employed for the calibration of the spectrometer. The broad and intense structure on the right corresponds to $N$-satellite X-ray lines originating from $M_{1,2,3} M_{4} N$ Coster-Kronig transitions. This structure could be well reproduced with one narrow and two broad juxtaposed Voigt profiles. All parameters were let free in the fit except the relative position and relative intensity of the line accounting for the asymmetry of the crystal response which were fixed at the same values as the ones obtained from the fits of the calibration lines. One could be tempted to assign the narrow profile at about $2285 \mathrm{eV}$ to the $M_{4}-N_{7}$ transition, the fitted energy shift of $3.8 \mathrm{eV}$ relative to the $M_{4}-N_{6}$ transition being consistent with the binding energy difference between the $4 f_{5 / 2}$ and $4 f_{7 / 2}$ levels. However, although we could not find any information in the literature about the strength of the $M_{4}-N_{7}$ transition, the latter is expected to be very weak because forbidden by the selection rule $\Delta j=0, \pm 1$. As this is in contradiction with the relatively strong intensity found for this line, the latter was assigned to the $N$-satellite structure of the $M_{4}-N_{6}$ transition.

\subsection{Satellite X-ray lines}

As a result of $L_{1} L_{2,3} M$ and $L_{1} L_{2,3} N$ Coster-Kronig transitions, $L_{1}^{-1}$ single-vacancy states may be transformed to $L_{2,3}^{-1} M^{-1}$ and $L_{2,3}^{-1} N^{-1}$ double-vacancy states. Such twohole states may also be created via $M$ - and $N$-shell shake processes $[27,28]$ as a consequence of the sudden change of the atomic potential following the $L$-shell photoionization. However, shakeup and shakeoff processes resulting from $L$-shell photoionization are characterized by very weak probabilities and can thus be neglected with respect to Coster-Kronig transitions. The radiative decay of doublevacancy states giving rise to satellite $\mathrm{X}$-ray lines, satellite structures are in general present in $L_{2}$ and $L_{3} \mathrm{X}$-ray spectra. On the contrary, in photoinduced $L_{1} \mathrm{X}$-ray spectra only diagram transitions corresponding to the radiative decay of single vacancy states are observed. The same 
holds for the $M_{4}$ and $M_{5}$ X-ray spectra in which strong $N$-satellite structures are usually observed as a result of $M_{1,2,3} M_{4,5} N$ Coster Kronig transitions (see, e.g., Fig. 3).

Satellite X-ray lines are slightly shifted in energy relative to their parent diagram lines. In general, the energy shifts are positive and decrease with the principal quantum number of the spectator vacancy. As a consequence, for $L_{2,3}$ transitions, satellites corresponding to $M$-shell spectator vacancies can be separated from their parent diagram lines, but satellites corresponding to $N$-shell spectator vacancies are usually not resolved, the energy shifts being smaller than the natural widths of the transitions. However, the energy shift of a satellite increasing with the principal quantum number of the transition electron, for $L_{2,3}$ transitions involving electrons from outer orbitals such as, e.g., $L_{2,3}-O$ transitions, $N$-satellites can again be resolved. Finally, energy shifts induced by spectator vacancies located in the outer $O$ - and $P$-shells are so small that the corresponding X-ray satellite lines have practically no noticeable effect on the line shapes of the parent diagram transitions.

For mercury, $L_{1} L_{2} M$ Coster-Kronig transitions are energetically forbidden but $L_{1} L_{2} N_{3-7}, L_{1} L_{3} N_{1-7}$ and $L_{1} L_{3} M_{4,5}$ transitions are allowed. The line shapes of $L_{2}$ and $L_{3}$ X-ray transitions may thus be affected by the unresolved $N$-satellites. In principle, $M$-satellites of $L_{3}$ transitions are less problematic for the data analysis since they can be resolved. However, due to the rather high density of lines characterizing $L$ X-ray spectra, the $M$-satellites may overlap with other close-lying diagram lines. In such cases the energies and widths of the overlapping diagram transitions may be affected by systematic errors if the $M$-satellites are not considered in the analysis. For these reasons we have determined the centroid energies and theoretical shapes of the $M$-satellites corresponding to the $L_{3}-M_{1,4,5}$ and $L_{3}-N_{1,4,5}$ transitions. Similar calculations were performed for the $N$-satellites of the $L_{2}-M_{1,4}$, $L_{2}-N_{1,4,6}, L_{3}-M_{1,4,5}$ and $L_{3}-N_{1,4,5,7}$ transitions. Results of these calculations were then employed in the data analysis to identify the observed transitions and to fit correctly the diagram transitions in the cases of unresolved $N$-satellites or overlapping $M$-satellites.

The line shape of a particular $j_{1} j_{2}(J) \rightarrow j_{3} j_{2}\left(J^{\prime}\right)$ satellite transition was constructed by computing numerically the sum of all components pertaining to the considered transition. In the above notation, $j_{1}$ and $j_{3}$ represent the angular momentum of the transition hole in the initial state (total angular momentum $J$ ) and final state (total angular momentum $J^{\prime}$ ), and $j_{2}$ the angular momentum of the spectator vacancy. The sum was calculated over all $\left(J, J^{\prime}\right)$ values permitted by the selection rule $\Delta J=0, \pm 1$. A Voigtian profile of energy $E$, Lorentzian width $\Gamma$ and relative intensity $I_{\text {rel }}$ was attached to each component. The energies of the different satellite components were derived from the experimental energy of the parent diagram transition and the energy shifts quoted in [29]. All components were given the same Gaussian and Lorentzian widths. For the Gaussian width the value assigned to the parent diagram transition was taken. The
Lorentzian width was approximated by:

$$
\Gamma=\Gamma_{j_{1}}+\Gamma_{j_{3}}+2 \Gamma_{j_{2}}
$$

where $\Gamma_{j_{1}}$ and $\Gamma_{j_{3}}$ are the widths of the subshells where the transition hole is located in the initial and final states and $\Gamma_{j_{2}}$ the width of the subshell where the spectator hole is located. The subshell widths were taken from [5]. The relative intensities of the components were assumed to be proportional to the relativistic electric-dipole X-ray emission for a double-hole-state transition [30]:

$$
I_{\text {rel }} \propto\left(2 J^{\prime}+1\right)\left(2 j_{1}+1\right)\left\{\begin{array}{ccc}
j_{3} & J^{\prime} & j_{2} \\
J & j_{1} & 1
\end{array}\right\} .
$$

The $6 j$-coefficients occurring in (6) were calculated using the formula and symmetry properties reported in [31]. It was found that most of the so-constructed $M$ and $N$-satellite profiles could be well reproduced by two juxtaposed Voigtians. In the fits the widths, positions and yield ratios of the two Voigtians were kept fixed at the values obtained from the calculations.

The relative intensities of the satellite lines which are equal to the ratios of double-to-single vacancy states were estimated using the radiationless transition probabilities reported recently by Santos et al. for the subshells $L_{1}$ [32] and $L_{2}$ [33], the fluorescence yields quoted in [34] and the relative intensities of the transitions $L_{1}-M_{2}, L_{2}-M_{4}$ and $L_{3}-N_{1}$ measured in the present work. The following results were obtained:

$$
\begin{aligned}
& \frac{I\left(L_{2}^{-1} N_{3-7}^{-1}\right)}{I\left(L_{2}^{-1}\right)}=0.06 \\
& \frac{I\left(L_{3}^{-1} N_{1-7}^{-1}\right)}{I\left(L_{3}^{-1}\right)}=0.15 \\
& \frac{I\left(L_{3}^{-1} M_{4,5}^{-1}\right)}{I\left(L_{3}^{-1}\right)}=0.23 .
\end{aligned}
$$

Rearrangement processes that modify the number of spectator vacancies prior to the $L \mathrm{X}$-ray emission were taken into account in these calculations. In particular, the Auger decay of the $M_{4,5}$ spectator vacancies into $N_{i}^{-1} N_{j}^{-1}$ double-vacancies was considered. This process leads to $L_{3}^{-1} N_{i}^{-1} N_{j}^{-1}$ triple-vacancy states, i.e., to second order $N$-satellites. Using the $M_{4,5} N_{i} N_{j}$ Auger rates reported by McGuire [35] and the fraction of $L_{3}^{-1} M_{4,5}^{-1}$ two-hole states given by (9), the following ratio was obtained for the triple-vacancy states:

$$
\frac{I\left(L_{3}^{-1} N_{1-7}^{-2}\right)}{I\left(L_{3}^{-1}\right)}=0.08 .
$$

Above estimates for the satellite yields were compared with the values obtained from the fits for the total satellite intensities. The comparisons were made using resolved and partially resolved satellites. More or less satisfactory agreements were found. However, some trend of the calculations to overestimate the intensities of the $M$-satellites 


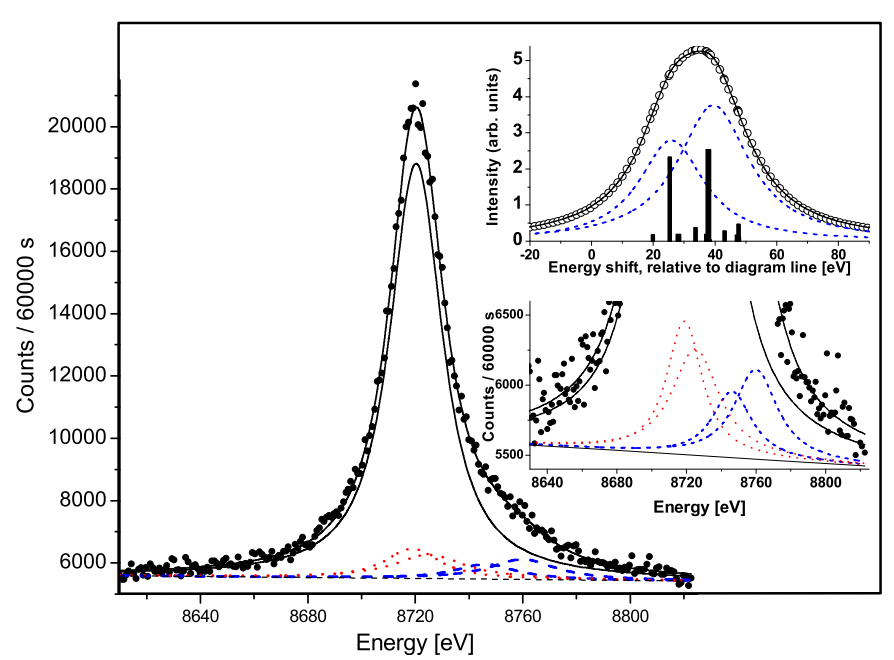

Fig. 4. (Color online) $\operatorname{Hg} L_{3}-M_{1}$ transition measured with the von Hamos spectrometer. The calculated $N$-satellites (dotted lines) and $M$-satellites (dashed lines) are shown enlarged in the bottom inset. The top inset shows the $M$-satellite components, the resulting satellite profile and the two Voigtians employed to reproduce the calculated shape. More details are given in the text.

was observed. For this reason, in most fits the total intensities of the satellites were let free and intensities fixed to above estimates were only employed in the case of nonconverging fits or when the satellite intensities from the fits were obviously wrong.

An example of the calculated $M$ and $N$-satellite profiles is presented in Figure 4 for the $L_{3}-M_{1}$ transition. The stick spectrum in the top inset represents the energies and relative intensities of the components of the $L_{3}^{-1} M^{-1} \rightarrow M_{1}^{-1} M^{-1}$ satellite transition, the open circles correspond to the sum of the Voigtians attached to the different components and the solid line stands for the fit of this sum with two Voigtians. The two pairs of Voigtians corresponding respectively to the $M$-satellite (dashed lines) and $N$-satellite (dotted lines) are represented enlarged in the bottom inset. As shown in the main figure the observed shape of the high-energy flank of the diagram transition could be well reproduced by the calculated $M$-satellite. One can also see that the $N$-satellite diminishes somewhat the fitted width of the diagram transition and has thus to be considered to obtain reliable results.

A further example is shown in Figure 5 representing the $L_{3}-N_{4,5}$ and $L_{1}-M_{3}$ transitions. One sees that in this case the two Voigtians corresponding to the $M$-satellite of the strong $L_{3}-N_{5}$ transition coincide accidentally in energy with the $L_{1}-M_{3}$ transition whose width is therefore significantly affected by the overlapping satellite. In the fit the $M$ and $N$-satellites of the $L_{3}-N_{4}$ and $L_{3}-N_{5}$ transitions were considered as well as the second order $N$-satellite of the $L_{3}-N_{5}$ transition. The line shape of this second order satellite being almost symmetric, the latter was fitted with a single Voigt profile (dashed-dotted line). The same holds for the $M$ and $N$-satellites of the weak $L_{3}-N_{4}$ transition. The seven Voigtians used to reproduce

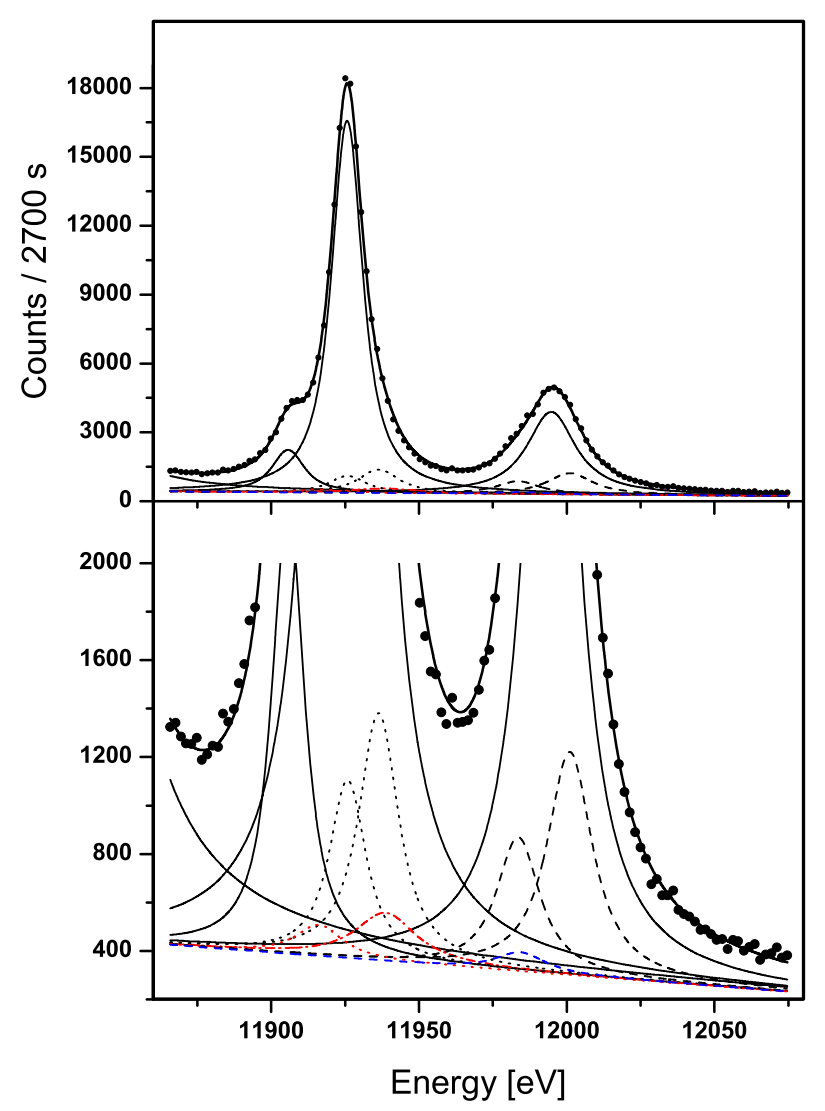

Fig. 5. (Color online) $\mathrm{Hg} L_{3}-N_{4,5}$ and $L_{1}-M_{3}$ transitions measured with the DuMond spectrometer. As shown, the $L_{1}-M_{3}$ diagram line is overlapping with the $M$-satellites (dashed lines) of the $L_{3}-N_{4,5}$ transitions which in turn are overlapping with their $N$-satellites (dotted lines). Details about the simulated satellites are shown in the bottom part of the figure. The weak dotted and dashed components correspond to the $N$ and $M$-satellites of the $L_{3}-N_{4}$ transition. The dasheddotted component represents the second order $N$-satellite of the $L_{3}-N_{5}$ transition. The higher background observed on the left side of the spectrum is due to the high-energy tail of the strong $L_{2}-M_{4}$ transition. The fitted tail is clearly visible in the bottom part of the figure.

the satellites are shown enlarged in the bottom part of the figure.

As the energy shifts for transitions from the $O$-shell are not quoted in [29], no simulation could be performed for the satellites of $L_{2,3}-O$ transitions. In [36], some data are available but they concern only the $L_{1}$-shell. Fortunately, no overlap between the $M$-satellites of the $L_{3}-O_{1}$ and $L_{3}-O_{5}$ transitions and other diagram transitions was observed so that the line shapes of these satellites were not needed in the data analysis. For the $N$-satellites of the $L_{2}-O_{1}, L_{2}-O_{4}$ and $L_{3}-O_{1}$ transitions, the energy shifts and widths of the two Voigtians were kept fixed at the values obtained from the fit of the resolved $L_{3}-O_{5}$ $N$-satellite, whereas the satellite intensities were either let free in the fits or fixed at the above theoretical estimates. The uncertainties related to these crude approximations were considered in the calculation of the errors reported for these transitions in Tables 1 and 3 (see next section). 


\section{Results and discussion}

\subsection{Transitions energies}

The energies of the $K, L$ and $M$ X-ray transitions observed in the present work are presented in Table 1. Experimental energies from Bearden [3] and theoretical values taken from [2] are also presented for comparison. Transition energies derived from the electron binding energies obtained in this work (see next subsection) are also quoted to point out which transitions deviate from the self-consistency principle.

As mentioned before, for mercury there are no other recent high-resolution measurements of X-ray transitions decaying core-vacancy states, except for the weak $L_{1}-M_{4,5}$ quadrupole transitions for which energies of $12457.05(52) \mathrm{eV}$ and $12547.60(29) \mathrm{eV}$ were reported [38]. Taking into consideration the small difference of the reference energy ( $\mathrm{Au} K \alpha_{1}$ transition) used in [38] $(68804.94 \mathrm{eV})$ and in the present work $(68804.50 \mathrm{eV})$, adjusted values of $12456.97(52) \mathrm{eV}$ and $12547.52(29) \mathrm{eV}$ are obtained, which are both in good agreement with the values quoted in Table $1(12457.10 \pm 0.54 \mathrm{eV}$ and $12547.68 \pm 0.31 \mathrm{eV})$. The energies found with the DuMond and von Hamos spectrometers for the $L_{2}-M_{4}$ transition differ by $0.46 \mathrm{eV}$ but the two results are consistent within the quoted errors. The total errors listed in Table 1 (first numbers in the brackets) include the fit errors which are also indicated (second numbers in the brackets). For the transitions observed with the von Hamos spectrometer, the total errors are generally bigger than those observed with the DuMond spectrometer for transitions of comparable energies. These bigger errors are mainly due to the uncertainties related to the energy calibration of the von Hamos spectrometer.

The comparison with Bearden's energies shows that about one third of the values are not consistent with present results within the combined errors, more than $15 \%$ of them being even not consistent within $3-\sigma$ errors. In particular, surprisingly strong deviations are observed for the $K-N_{3}(+18.9 \mathrm{eV}), L_{1}-M_{4}(-11.6 \mathrm{eV}), L_{1}-M_{5}$ $(+12.6 \mathrm{eV}), L_{1}-N_{5}(-9.8 \mathrm{eV})$ and $L_{3}-N_{7}(+9.8 \mathrm{eV})$ transitions. It can be also noted that the errors of the present transition energies are in average about three times smaller than those of Bearden. The comparison with the theoretical energies reported in [2] shows on the contrary an excellent agreement since all quoted values are consistent with present results. The errors listed in [2] for the theoretical transition energies could even be somewhat overestimated since the average deviations between the theoretical values and present results are about 2.5 times smaller than the reported theoretical uncertainties. Five kinds of contributions were considered in these calculations, namely the finite size of the atomic nucleus, different relativistic effects (corrections to the Coulomb energy, magnetic and retardation energy), the Coulomb and Breit correlation, several radiative (QED) corrections and the Auger shift. More details about the theoretical procedures used to perform these calculations can be found in [39].
A good agreement is also observed between the transition energies directly measured and those derived from the binding energies. Actually, Table 1 shows that only for the $L_{2}-M_{1}$ and $L_{3}-N_{4}$ transitions the two energies are not consistent. For the first transition, the measured energy is smaller by $1.45 \mathrm{eV}$ than the one deduced from the binding energy difference, whereas the combined error of the two energies is $\pm 0.78 \mathrm{eV}$. No explanation was found for that discrepancy. For the $L_{3}-N_{4}$ transition, the measured energy is bigger by $0.61 \mathrm{eV}$ and the combined error is $\pm 0.28 \mathrm{eV}$. In this case, the explanation for the discrepancy resides probably in the difficulties encountered to fit precisely the weak $L_{3}-N_{4}$ transition, the latter being poorly separated from the stronger close-lying $L_{3}-N_{5}$ transition (see Fig. 5). For these two inconsistent transition energies, the values derived from the binding energy differences are probably more reliable. They are also closer to the theoretical predictions than the measured ones. Finally, one would like to point out that the errors of the energies computed from the binding energies are smaller than those of the measured energies and that they do not depend on the intensity of the considered X-ray line. This method presents thus the advantage to provide also precise energies in the case of weak transitions. The errors quoted in Table 1 for the transition energies derived from the binding energy differences were computed by means of the following relation:

$$
\Delta E_{i j}= \pm \sqrt{\sigma_{i i}^{2}+\sigma_{j j}^{2}-2 \sigma_{i j}}
$$

where $\Delta E_{i j}$ represents the error on the energy of the transition $i \rightarrow j$, and $\sigma_{i i}^{2}, \sigma_{j j}^{2}$ and $\sigma_{i j}$ are diagonal variance elements, respectively off-diagonal covariance elements, of the error matrix related to the least-squares fit method employed to compute the binding energies.

\subsection{Binding energies}

As the energy of an X-ray transition is equal to the difference of the binding energies of the two atomic levels involved in that transition, a linear system of simultaneous equations could be deduced from the transition energies listed in Table 1. The system was solved by means of a least-squares-fitting method, using the total errors on the transition energies to weight the different equations. For the binding energies of the subshells $N_{1}$ to $N_{7}$ and $\mathrm{O}_{2}$ to $\mathrm{O}_{5}$, reliable XPS data do exist. In addition, these binding energies are small relative to the energies of the transitions in which the above subshells are involved. Thus, with the least-squares-fit method these values cannot be determined with high precision. For these reasons, the binding energies of these levels were not used as variables but replaced in the corresponding equations by their known values. For the latter, the average values of the binding energies reported in $[4,7]$ were taken, using the half-differences between the two values as uncertainty estimates. For the subshell $O_{1}$, however, no value is quoted in [7] for liquid $\mathrm{Hg}$ (a binding energy of $134 \pm 5 \mathrm{eV}$ is given but for the $\mathrm{Hg}$ vapor) and the value reported by 
Table 2. Binding energies in eV of the levels $K$ to $M_{5}$ and $O_{1}$. Results obtained in the present work are compared to compiled experimental data [41,42] and to predictions from theoretical calculations [43-45].

\begin{tabular}{|c|c|c|c|c|c|}
\hline Level & Present work & Bearden and Burr [41] & Storm and Israel [42] & Polasik [43] & $\begin{array}{l}\text { Huang et al. [44] } \\
\text { Chen et al. [45] }\end{array}$ \\
\hline$K$ & $83102.75(47)$ & $83102.3(8)$ & 83102 & 83110.0 & $\begin{array}{l}83101.0 \\
83105.6^{*}\end{array}$ \\
\hline$L_{1}$ & $14843.19(15)$ & $14839.3(1.0)$ & 14842 & 14859.0 & $\begin{array}{c}14857.6 \\
14858.7^{*}\end{array}$ \\
\hline$L_{2}$ & 14209.61(11) & $14208.7(7)$ & 14209 & 14217.0 & $\begin{array}{c}14213.9 \\
14214.1^{*}\end{array}$ \\
\hline$L_{3}$ & $12284.60(11)$ & $12283.9(4)$ & 12283 & 12292.3 & $\begin{array}{c}12289.8 \\
12289.2^{*}\end{array}$ \\
\hline$M_{1}$ & $3562.55(31)$ & 3561.6(1.1) & 3562 & 3578.3 & 3583.7 \\
\hline$M_{2}$ & $3280.10(23)$ & $3278.5(1.3)$ & 3280 & 3292.0 & 3291.9 \\
\hline$M_{3}$ & $2848.53(24)$ & $2847.1(4)$ & 2847 & 2861.0 & 2860.1 \\
\hline$M_{4}$ & $2386.23(9)$ & $2384.9(3)$ & 2385 & 2393.9 & 2393.1 \\
\hline$M_{5}$ & $2295.55(11)$ & $2294.9(3)$ & 2295 & 2303.5 & 2302.5 \\
\hline$O_{1}$ & $121.81(26)$ & $120.3(1.3)$ & 121 & & 133.6 \\
\hline
\end{tabular}

Fuggle and Martensson (127 eV) [4] looks dubious because it is inconsistent with the results (about $120 \mathrm{eV}$ ) from other sources (e.g. [41,42]). The binding energy of the $5 s_{1 / 2}$ level was therefore used as an additional free fitting parameter. Thus the system consisted of 31 linear equations with 10 unknowns, namely the electron binding energies of the $K$-shell and $L_{1-3}, M_{1-5}$ and $O_{1}$-subshells. The so-obtained binding energies are presented in Table 2. Quoted uncertainties originate from the error matrix of the least-squares-fit. They correspond to relative uncertainties of about $5 \mathrm{ppm}$ for the $K$-shell, $10 \mathrm{ppm}$ for the $L$-subshells and $80 \mathrm{ppm}$, respectively $40 \mathrm{ppm}$, for the $M_{1-3}$ and $M_{4,5}$-subshells. For the $O_{1}$-subshell a satisfactory precision of about $0.3 \mathrm{eV}(0.2 \%)$ was obtained. The biggest relative uncertainties are found for the $M_{1-3}$ and $O_{1}$-subshells because these levels occur only in two transitions, whereas other subshells are involved in a number of transitions varying from three for the $M_{5}$-subshell up to ten for the $L_{3}$-subshell.

We tried therefore to extend the measurements to additional $M_{1-3} \mathrm{X}$-ray lines. The energy regions corresponding to the $M_{1}-N_{3}, M_{1}-O_{3}, M_{2}-N_{4}, M_{2}-O_{4}, M_{3}-N_{5}$, $M_{3}-O_{1}$ and $M_{3}-O_{5}$ transitions were explored. The measurements were performed with the ADP (101) crystal in second order of diffraction, except for the $M_{3}-N_{5}$ transition which was measured in first order. Although some of these transitions were observed successfully for Th and U with the same von Hamos spectrometer [46], for $\mathrm{Hg}$ no significant intensity could be found above the background at the expected positions of the transitions. This is due to the cumulative effects of several factors such as the poorer fluorescence yields of the $M$-subshells of $\mathrm{Hg}$ as compared to those of Th and $\mathrm{U}$, the bigger self-absorption of the $\mathrm{Hg} M \mathrm{X}$-rays in the target and to their additional attenuation by the Kapton ${ }^{\mathrm{TM}}$ window.

In Table 2, present binding energies are compared to experimental results from Bearden and Burr [41], semiempirical values from Storm and Israel [42] and theoretical predictions from different sources [43-45]. Actually, compilations of experimental electron binding energies that are more recent than the one of Bearden and Burr do exist but data given in these papers for core levels of mid-heavy and heavy elements are still those from Bearden and Burr so that we have preferred to quote the original reference. For instance, for $\mathrm{Hg}$ the binding energies listed in [47] coincide with Bearden and Burr's values, except those for the outer $N$ - and $O$-shells that were taken from [4]. From the examination of the table one sees first that present binding energies are systematically higher than those of Bearden and Burr. The biggest discrepancy is observed for the $L_{1}$-subshell for which the deviation amounts to $3.9 \mathrm{eV}$, which is about 4 times more than the uncertainty quoted by Bearden and Burr for this level. For the other levels, the average deviation is approximately $1 \mathrm{eV}$ whereas the average uncertainty is about $0.8 \mathrm{eV}$. A better agreement is found with Storm and Israel's values, the average deviation being $0.9 \mathrm{eV}$ and the average uncertainty $1 \mathrm{eV}$. In particular, for the $L_{1}$-subshell the energy quoted by Storm and Israel is almost consistent with ours. It can be also noted that the values given by Bearden and Burr and Storm and Israel for the binding energy of the $O_{1}$-subshell are both consistent with our result, whereas the more recent XPS value from [4] used in the above-mentioned recent compilation [47] is significantly bigger $(127 \mathrm{eV})$.

As shown in Table 2, the binding energies predicted by theory are all higher by $5-20 \mathrm{eV}$ than present experimental results, except for the $K$-shell for which a satisfactory agreement is found, at least for the values taken from [44,45]. The largest deviations are observed for the $M_{1}$-subshell $(20 \mathrm{eV})$ and the $L_{1}$-susbshell $(15 \mathrm{eV})$. The multiconfiguration Dirac-Fock (MCDF) [43] and Dirac-Hartree-Fock-Slater (DHFS) [44,45] calculations give similar results within $1-3 \mathrm{eV}$ except for the $K$-shell and the $M_{1}$-subshell. The MCDF value for the $K$-shell is indeed significantly bigger than the experimental and DHFS theoretical values, whereas for the $M_{1}$-subshell an opposite trend is observed. In both MCDF and DHFS calculations the magnetic interaction energy, retardation energy, vacuum polarization correction and higher-order QED corrections were considered. 
The MCDF calculations were performed, using the modified special average-level version (MSAL) [48] of the GRASP code [49]. The DHFS calculations from [45] differ from the earlier ones [44] by the use of a more accurate expression for the Breit energy, a Fermi instead of uniform distribution of the nuclear charge and a screened self-energy correction. The improvements brought by the new calculations are not directly visible in Table 2 but the transition energies provided by the new calculations agree significantly better with the experimental results. For instance, for the $K \alpha_{1}$ and $K \alpha_{2}$ transitions, deviations of $-1.7 \mathrm{eV}$ and $-1.6 \mathrm{eV}$ are found with respect to the experimental values obtained in the present work (see Tab. 1 third column), whereas the corresponding deviations for the old DHFS calculations are $-6.9 \mathrm{eV}$ and $-5.9 \mathrm{eV}$, respectively. Note that the same comparison made with the MCDF predictions provides also a good agreement, the deviations being only $-0.4 \mathrm{eV}$ and $-1.4 \mathrm{eV}$, respectively. A further comparison with the theoretical binding energies quoted in [2] shows that the calculations overestimate the binding energies of the $K$-shell and the $L_{1-3}$-subshells by $8.5,7.6,7.3$ and $7.7 \mathrm{eV}$, respectively. However, by correcting the zero of the theoretical energy scale by $-7.8 \mathrm{eV}$, the adjusted edge energies are found to be fully consistent with present results.

\subsection{Transition widths}

The widths of the measured transitions are presented in Table 3 where they are compared to existing experimental and theoretical data. As in the fits of the transitions $K-N_{2}, L_{1}-N_{4}$ and $L_{1}-O_{2}$ the linewidths of the latter could be let free, whereas their energies had to be kept fixed, these transitions are quoted in Table 3 but not in Table 1 . The values listed in the third column were derived from the level widths obtained by the least-squaresfit method discussed in the next subsection. As shown, these values are all in good agreement with the measured transition widths, except the $L_{1}-N_{4}$ transition for which there is a deviation $(1.9 \mathrm{eV})$ that is somewhat bigger than the combined error $(1.6 \mathrm{eV})$. This discrepancy is probably due to the small intensity and poor separation of the $L_{1}-N_{4,5}$ quadrupole doublet which made the width determination of the weaker component less reliable.

As mentioned in the introduction, for $\mathrm{Hg}$ experimental information concerning the natural linewidths of X-ray lines is scarce. In addition, existing data are rather old. For the $K \alpha_{1,2}$ transitions, widths of $68.5 \mathrm{eV}$ and $64.0 \mathrm{eV}$, respectively, were reported $[50-52]$ that are $5-10 \%$ bigger than present results but consistent within the rather large uncertainties quoted by the authors. The values of $68.20 \mathrm{eV}$ and $64.75 \mathrm{eV}$ given by Salem and Lee [54] for the same transitions were derived from a least-squares-fit to existing experimental data till 1976 . For the $K \beta_{1,3}$ transitions, the widths given by Salem and Lee are also bigger than ours but consistent within the indicated errors $(10 \%)$. Interpolation values obtained from the widths of the $K \alpha_{1,2}$ transitions of $\mathrm{Au}$ and $\mathrm{Pb}$ from [58] are also quoted. In this case, an excellent agreement with our results is observed.

For $L$ X-rays, available experimental data are even more rare than for $K$ X-rays. In Salem and Lee's tables values are reported only for the seven strongest $L$ transitions. Quoted values are consistent with present results if one considers the uncertainties assumed in these tables. The mean value of the deviations relative to present results is about $8 \%$, the strongest differences being observed for the transitions $L_{1}-M_{2}(-2.9 \mathrm{eV}), L_{1}-M_{3}(-1.2 \mathrm{eV})$ and $L_{2}-N_{4}(+0.80 \mathrm{eV})$. In the region $70 \leq Z \leq 83$, Salem and Lee's least-squares-fit values for $L$ transition widths are based mainly on two old works, those of Cooper [59] and Williams [60]. Unfortunately in these two papers no data is reported for $\mathrm{Hg}$. Values quoted in Table 3 were thus obtained by interpolation from the widths given for $\mathrm{Au}$ and Tl. The eight transition widths derived from Cooper's work are smaller than ours by $5-15 \%$. This is probably due to the fact that the Lorentzian widths given in this paper were obtained by subtracting the instrumental broadening from the observed widths and not by deconvolution. In the still older paper of Williams, only the widths of the four strongest transitions $\left(L \alpha_{1}, L \beta_{1}, L \beta_{2}\right.$ and $\left.L \gamma_{1}\right)$ were determined. The measurements were performed with a double-flat crystal spectrometer, whose resolution depended on the divergence of the incident X-ray beam and finite resolving power of the employed calcite crystals. The influence of the beam divergence was assumed to be negligibly small and the crystal resolving power was not well known so that no correction was made for the instrumental broadening. This explains probably the systematic bigger values obtained by Williams with respect to present results. However, we would like to point out that, despite this systematic deviation, Williams' widths are consistent with ours.

The transition widths reported in the last column of Table 3 were derived from semi-empirical and theoretical atomic level widths. As shown, the widths of the $K \alpha_{1,2}$ transitions obtained from the semi-empirical level widths quoted by Krause and Oliver [53] agree well with present results. The same holds for the $K \alpha_{1,2}$ widths obtained from the theoretical widths $\Gamma_{K}[55]$ and $\Gamma_{L_{2,3}}$ [56] calculated by Chen et al. Theoretical predictions for the $L_{1}$ transition widths are on the contrary systematically bigger by about $20 \%$ than present experimental values. The same trend is observed for the transitions involving $M_{1}$ holes, whereas for $L_{2}$ and $L_{3}$ transitions involving $M_{4,5}$ vacancies in the final states, a satisfactory agreement is found. This observation that theory overestimates the transition widths when $2 s$ [38] or $3 s$ [64] vacancies are present in the initial or final state results from the difficulty of calculations to provide reliable predictions for the Coster-Kronig rates. In general, the latter are indeed overestimated by theory. For instance, theoretical $L_{1}$ Coster-Kronig widths of $14.5 \mathrm{eV}, 11.2 \mathrm{eV}$ and $10.7 \mathrm{eV}$ are given in [65] (interpolation between the values quoted for $\mathrm{Au}$ and $\mathrm{Bi}$ ), [66] and [32], respectively. The theoretical Auger widths from the same sources are $2.5 \mathrm{eV}$, $2.0 \mathrm{eV}$ and $2.1 \mathrm{eV}$, i.e., $2.2 \mathrm{eV}$ in average. Subtracting 
Table 3. Linewidths in $\mathrm{eV}$ of the measured $\mathrm{Hg}$ transitions. The values quoted in the third column were derived from the widths of the levels $K-N_{5}$ and $O_{1}$ obtained by the least-squares fit method (see Sect. 4.4). The notation 61.3(2.2/7) means $61.3 \pm 2.2 \mathrm{eV}$ with an included statistical error from the fit of $\pm 0.7 \mathrm{eV}$.

\begin{tabular}{|c|c|c|c|c|}
\hline Transition & Present work & $\begin{array}{c}\text { Calculated from present } \\
\text { level widths }\end{array}$ & $\begin{array}{l}\text { Experimental data } \\
\text { from other sources }\end{array}$ & $\begin{array}{c}\text { Semi-empirical and } \\
\text { theoretical data }\end{array}$ \\
\hline$K-L_{2}$ & $61.3(2.2 / 7)^{a}$ & $60.6(9)$ & $\begin{array}{c}68.5(10.3)^{b} \\
68.2^{d} \\
61.3(3.5)^{f}\end{array}$ & $\begin{array}{c}60.8(1.8)^{c} \\
60.5^{e}\end{array}$ \\
\hline$K-L_{3}$ & $61.2(2.3 / 6)^{a}$ & $61.2(9)$ & $\begin{array}{c}64.0(9.6)^{b} \\
64.7^{d} \\
60.4(1.1)^{f}\end{array}$ & $\begin{array}{c}60.1(1.8)^{c} \\
60.5^{e}\end{array}$ \\
\hline$K-M_{2}$ & $65.2(3.3 / 1.3)^{a}$ & $66.9(9)$ & $68.9^{d}$ & $68.4^{e}$ \\
\hline$K-M_{3}$ & $61.9(3.3 / 9)^{a}$ & $63.0(9)$ & $65.7^{d}$ & $65.0^{e}$ \\
\hline$K-N_{2}$ & $62.7(4.2 / 2.4)^{a}$ & $64.3(1.0)$ & & \\
\hline$K-N_{3}$ & $64.5(3.7 / 1.4)^{a}$ & $61.9(9)$ & & \\
\hline$L_{1}-M_{2}$ & $22.6(3 / 2)^{a}$ & $22.6(2)$ & $\begin{array}{c}19.7^{d} \\
20.1(8)^{g}\end{array}$ & $28.1^{e}$ \\
\hline$L_{1}-M_{3}$ & $18.6(3 / 2)^{a}$ & $18.6(1)$ & $\begin{array}{c}17.4^{d} \\
17.9(1.2)^{g}\end{array}$ & $24.7^{e}$ \\
\hline$L_{1}-M_{4}$ & $13.3(1.4 / 1.3)^{a}$ & $12.9(4)$ & & $16.9^{e}$ \\
\hline$L_{1}-M_{5}$ & $12.9(1.0 / 9)^{a}$ & $12.8(4)$ & & $16.9^{e}$ \\
\hline$L_{1}-N_{2}$ & $20.0(5 / 5)^{a}$ & $19.9(3)$ & $17.2(8)^{g}$ & \\
\hline$L_{1}-N_{3}$ & $17.5(4 / 3)^{a}$ & $17.6(3)$ & $15.6(6)^{g}$ & \\
\hline$L_{1}-N_{4}$ & $13.5(1.5 / 1.4)^{a}$ & $15.4(4)$ & & \\
\hline$L_{1}-N_{5}$ & $15.5(1.2 / 1.1)^{a}$ & $14.9(4)$ & & \\
\hline$L_{1}-O_{2}$ & $19.3(7 / 7)^{a}$ & & & \\
\hline$L_{1}-O_{3}$ & $18.6(6 / 6)^{a}$ & & & \\
\hline$L_{2}-M_{1}$ & $21.4(5 / 5)^{h}$ & $21.5(1)$ & & $23.9^{e}$ \\
\hline$L_{2}-M_{4}$ & $\begin{array}{c}8.39(14 / 4)^{a} \\
8.89(30 / 13)^{h}\end{array}$ & $8.44(9)$ & $\begin{array}{c}8.70^{d} \\
7.6(2)^{g} \\
8.63(26)\end{array}$ & $8.1^{e}$ \\
\hline$L_{2}-N_{1}$ & $15.1(7 / 7)^{a}$ & $14.8(3)$ & & \\
\hline$L_{2}-N_{4}$ & $11.0(2 / 1)^{a}$ & $10.9(1)$ & $\begin{array}{c}11.8^{d} \\
10.1(3)^{g} \\
11.8(6)^{i}\end{array}$ & \\
\hline$L_{2}-N_{6}$ & $6.82(61 / 56)^{a}$ & & & \\
\hline$L_{2}-O_{1}$ & $14.8(1.0 / 9)^{a}$ & $15.0(4)$ & & \\
\hline$L_{2}-O_{4}$ & $7.46(36 / 27)^{a}$ & & $7.1(7)^{g}$ & \\
\hline$L_{3}-M_{1}$ & $22.1(1 / 1)^{h}$ & $22.1(1)$ & & $23.9^{e}$ \\
\hline$L_{3}-M_{4}$ & $9.20(21 / 16)^{h}$ & $9.00(11)$ & $8.80^{d}$ & $8.2^{e}$ \\
\hline$L_{3}-M_{5}$ & $8.87(16 / 4)^{h}$ & $8.92(10)$ & $\begin{array}{c}8.10^{d} \\
9.04(18)^{i}\end{array}$ & $8.2^{e}$ \\
\hline$L_{3}-N_{1}$ & $15.3(4 / 4)^{a}$ & $15.4(3)$ & & \\
\hline$L_{3}-N_{4}$ & $11.5(3 / 2)^{a}$ & $11.5(1)$ & & \\
\hline$L_{3}-N_{5}$ & $11.0(2 / 1)^{a}$ & $11.0(1)$ & $\begin{array}{c}10.4^{d} \\
10.2(4)^{g} \\
11.8(6)^{i}\end{array}$ & \\
\hline$L_{3}-N_{7}$ & $5.86(66 / 63)^{a}$ & $6.77(13)$ & & \\
\hline$L_{3}-O_{1}$ & $15.7(7 / 7)^{a}$ & $15.6(4)$ & & \\
\hline$L_{3}-O_{5}$ & $7.53(20 / 13)^{a}$ & & & \\
\hline$M_{4}-N_{6}$ & $2.79(20 / 2)^{h}$ & $2.87(11)$ & & $2.95^{j}$ \\
\hline$M_{5}-N_{7}$ & $2.84(19 / 2)^{h}$ & $2.77(11)$ & & $2.81^{j}$ \\
\hline
\end{tabular}

${ }^{a}$ DuMond spectrometer. For details see Table 1 .

${ }^{b}$ Nelson et al. [50-52].

${ }^{c}$ Krause and Oliver [53].

${ }^{d}$ Salem and Lee [54] (errors vary from $10 \%$ for the strongest $K$ lines to $40 \%$ for the weakest $L$ lines).

e Calculated from the widths $\Gamma_{K}, \Gamma_{L 1,2,3}, \Gamma_{M 1,2,3}$ and $\Gamma_{M 4,5}$ reported by Chen et al. in [55], [56], [40] and [57], respectively.

${ }^{f}$ Derived by interpolation from the widths reported for $\mathrm{Au}$ and $\mathrm{Pb}$ by Kessler et al. [58].

${ }^{g}$ Derived by interpolation from the widths reported for $\mathrm{Au}$ and $\mathrm{Tl}$ by Cooper [59].

${ }^{h}$ Von Hamos spectrometer. For details see Table 1.

${ }^{i}$ Derived by interpolation from the widths reported for $\mathrm{Au}$ and $\mathrm{Tl}$ by Williams [60].

${ }^{j}$ Derived by interpolation from the widths $\Gamma_{M 4,5}$ and $\Gamma_{N 6,7}$ reported for Au and Bi by McGuire in [61] and [62,63], respectively. 
the latter average Auger width and the radiative width of the $2 s$ level reported by Scofield $(1.2 \mathrm{eV})[26]$ from the experimental width $(10.5 \mathrm{eV})$ of the subshell $L_{1}$ quoted by Campbell and Papp [5], one obtains a Coster Kronig width of $7.1 \mathrm{eV}$ which is markedly smaller than the three above-mentioned theoretical values. Finally, the theoretical widths of the transitions $M_{4}-N_{6}$ and $M_{5}-N_{7}$ deduced from the widths of the corresponding levels calculated by McGuire [61-63] are in fair agreement with our experimental values.

\subsection{Atomic level widths}

Using the widths of the measured transitions, we were able to determine the natural widths of the $K$-shell, $L_{1}$ to $N_{5}$ and $O_{1}$-subshells by means of a similar least-squares-fitmethod as the one employed for the determination of the binding energies. In this case the system consisted of thirty equations with the widths of the above mentioned fifteen levels as unknowns. The widths of the $N_{6,7}$-subshells were kept fixed in the fit at the values $(0.33 \mathrm{eV}$ and $0.31 \mathrm{eV})$ given by Campbell and Papp [5]. As each of the levels $\mathrm{O}_{2}$ to $\mathrm{O}_{5}$ occur in a single transition, they were not included in the least-squares-fit calculations. The level widths obtained with this method are presented in Table 4 where they are compared to the recommended values of Campbell and Papp [5] as well as to available experimental, semi-empirical and theoretical results from various sources. Errors on present results correspond to the diagonal elements of the error matrix associated to the least-squares-fit method. They vary from about $1 \%$ ( $M_{1}$-subshell) to about $7 \%\left(N_{3}\right.$-subshell $)$. The precision depends on the number of transitions in which the considered level is involved, the uncertainties of the widths of these transitions and on the relative value of the level width with respect to the widths of the associated transitions.

The comparison with Campbell and Papp's values shows a fair agreement for most levels, except for the $M_{2}, N_{2}$ and $N_{3}$ ones for which significant differences of $2.4 \mathrm{eV}, 3.1 \mathrm{eV}$ and $1.9 \mathrm{eV}$, respectively, are observed. The widths of the three levels quoted by Campbell and Papp are smaller than ours by about $30 \%$. For the $N_{4,5}$ levels, a similar trend is observed but the deviations are smaller (15\%). However, despite the observed discrepancies, we are inclined to believe that present results for the $M_{2}$ and $N_{2-5}$ levels are reliable for the following reasons. The $M_{2}$ level width of $\mathrm{Hg}$ recommended by Campbell and Papp was determined by interpolation from the widths of the transitions $L_{1}-M_{2}$ of $\mathrm{Au}$ and $\mathrm{Tl}$ reported by Cooper [59], using for the width of the $2 s$ level their recommended value of $10.5 \mathrm{eV}$. The so-obtained $M_{2}$ width was then increased by $0.2 \mathrm{eV}$ to account for the too large correction made by Cooper for the instrumental broadening. Since Campbell and Papp's value for the width of the $L_{1}$-subshell is close to the one obtained in the present work, the discrepancy observed for the $M_{2}$ - subshell originates from the width of the $L_{1}-M_{2}$ transition quoted by
Cooper which is certainly too small, as discussed in the preceding subsection.

For the $N_{2,3}$-subshells of elements above $Z=70$, there are no recent XES data except for actinides and only a limited number of XPS data. In addition, the XPS data evince a considerable scatter. For this reason Campbell and Papp have deduced their recommended $N_{2,3}$ level widths from least-squares-fit curves that run monotonically through the existing XPS data and join smoothly to modern XES data that are available for Th [46] and U [11]. On the low-Z side, the curves were anchored to the Xe $N_{2,3}$ widths obtained from many-body theory (MBT) calculations [68]. In our opinion, it is thus possible that the $N_{2,3}$ level widths quoted by Campbell and Papp for that region of the periodic table are questionable and characterized by bigger errors than the quoted ones. Furthermore, comparing the widths reported by Cooper [59] for the transitions $L_{1}-M_{2,3}, L_{2}-M_{4}, L_{2}-N_{4}, L_{2}-O_{4}$ and $L_{3}-N_{5}$ with the results obtained in this work (see Tab. 3), an average ratio of $0.92 \pm 0.03$ is found for the two sets of values. Using this ratio to adjust the widths of the transitions $L_{1}-N_{2,3}$ given by Cooper, new values of $18.6 \pm 1.0 \mathrm{eV}$ and $16.9 \pm 0.8 \mathrm{eV}$ are found for the two transitions widths. Subtracting then from the latter the $L_{1}$ level width recommended by Campbell and Papp $(10.5 \pm 2.0 \mathrm{eV})$, one obtains $N_{2,3}$ widths of $8.1 \pm 2.2 \mathrm{eV}$ and $6.4 \pm 2.1 \mathrm{eV}$ that are still smaller than our results but consistent with them.

For the $N_{4,5}$-subshells several difficulties were also encountered by Campbell and Papp. Actually, in the region $70 \leq Z \leq 83$, XPS data are more numerous and cluster quite closely but their results disagree with values derived from measurements of the $L_{2}-N_{4}$ and $L_{3}-N_{5}$ transitions $[59,69]$ which lie $1.5 \mathrm{eV}$ to $3 \mathrm{eV}$ higher. The small number of available data from Auger electron spectroscopy (AES) [70] lie also high relative to the XPS trend. As these XES and AES data are pretty old, Campbell and Papp renounced to use them and preferred to employ the XPS data. However, they mentioned that the sodetermined widths could be somewhat too small and that it would be therefore desirable to cross-check them with a modern set of measurements of the $L_{2}-N_{4}$ and $L_{3}-N_{5}$ X-ray linewidths.

Existing experimental information for the level widths of $\mathrm{Hg}$ is scarce. Data were found only for the $L, N$ and $O$-subshells. The $L$-subshells widths were determined by Keski-Rahkonen et al. from X-ray absorption spectroscopy (XAS) measurements [6]. The obtained level widths are in agreement with present results for the $2 p$ levels but in strong disagreement for the $2 s$ level for which a value smaller by $4 \mathrm{eV}$ is reported in that work. For the $N$ and $O$-subshells XPS data were published by Svensson et al. [7]. However, quoted level widths were not corrected for the instrumental broadening and no indication is given for the uncertainties attached to the quoted values. Nevertheless, one sees in Table 4 that a satisfactory agreement with present results is observed for the $N_{1}$ and $N_{2}$ levels, whereas for the $N_{3-5}$ levels, the XPS widths are $0.5-1.5 \mathrm{eV}$ smaller than present results. Note that Svensson's values for the $N_{3-5}$ levels are close to 
Table 4. Level widths in eV of the subshells $K$ to $N_{5}$ and $O_{1}$. Results obtained in the present work are compared to available experimental [5-7], semi-empirical [53] and theoretical data [35,40,55-57,62,63].

\begin{tabular}{|c|c|c|c|c|}
\hline Level & Present work & Campbell and Papp [5] & $\begin{array}{l}\text { Experimental data } \\
\text { from other sources }\end{array}$ & $\begin{array}{l}\text { Semi-empirical and } \\
\text { theoretical data }\end{array}$ \\
\hline$K$ & $54.7(9)$ & $54.8(4.9)$ & & $\begin{array}{c}54.6(1.6)^{a} \\
54.8^{b}\end{array}$ \\
\hline$L_{1}$ & $10.3(4)$ & $10.5(2.0)$ & $6.4(2.0)^{c}$ & $\begin{array}{c}11.3(1.7)^{a} \\
14.5^{b}\end{array}$ \\
\hline$L_{2}$ & $5.90(13)$ & $5.69(57)$ & $6.0(1.0)^{c}$ & $\begin{array}{c}6.17(62)^{a} \\
5.7^{b}\end{array}$ \\
\hline$L_{3}$ & $6.46(12)$ & $5.71(57)$ & $5.8(8)^{c}$ & $\begin{array}{c}5.50(44)^{a} \\
5.7^{b}\end{array}$ \\
\hline$M_{1}$ & $15.6(2)$ & $15.1(2.0)$ & & $\begin{array}{l}18.2^{b} \\
21.1^{d}\end{array}$ \\
\hline$M_{2}$ & $12.2(5)$ & $9.8(1.8)$ & & $\begin{array}{l}13.6^{b} \\
14.7^{d}\end{array}$ \\
\hline$M_{3}$ & $8.25(48)$ & $8.6(1.6)$ & & $\begin{array}{l}10.2^{b} \\
9.7^{d}\end{array}$ \\
\hline$M_{4}$ & $2.54(11)$ & $2.28(23)$ & & $\begin{array}{l}2.45^{b} \\
2.82^{d}\end{array}$ \\
\hline$M_{5}$ & $2.46(11)$ & $2.28(23)$ & & $\begin{array}{l}2.45^{b} \\
2.68^{d}\end{array}$ \\
\hline$N_{1}$ & $8.93(29)$ & $8.8(9)$ & $\sim 10^{e}$ & $\begin{array}{l}15.4^{d} \\
7.75^{f}\end{array}$ \\
\hline$N_{2}$ & $9.61(55)$ & $6.55(80)$ & $\sim 9^{e}$ & $\begin{array}{l}14.1^{d} \\
7.87^{f}\end{array}$ \\
\hline$N_{3}$ & $7.22(50)$ & $5.3(8)$ & $5.5^{e}$ & $\begin{array}{l}12.6^{d} \\
4.40^{f}\end{array}$ \\
\hline$N_{4}$ & $5.05(17)$ & $4.0(5)$ & $4.4^{e}$ & $\begin{array}{l}8.5^{d} \\
5.37^{f}\end{array}$ \\
\hline$N_{5}$ & $4.59(15)$ & $3.85(50)$ & $4.0^{e}$ & $\begin{array}{c}8.2^{d} \\
5.22^{f}\end{array}$ \\
\hline$O_{1}$ & $9.11(41)$ & & & \\
\hline
\end{tabular}

${ }^{a}$ Krause and Oliver [53].

${ }^{b}$ Chen et al., $\Gamma_{K}[55], \Gamma_{L 1,2,3}[56], \Gamma_{M 1,2,3}[40]$ and $\Gamma_{M 4,5}[57]$.

${ }^{c}$ Keski-Rahkonen et al. [6] (XAS measurements).

${ }^{d}$ Estimated from an interpolation of the widths $\Gamma_{M 1-M 5}$ and $\Gamma_{N 1-N 5}$ reported for Au and Bi by McGuire in [61] and [62,63], respectively.

${ }^{e}$ Svensson et al. [7] (XPS measurements, results not corrected for spectrometer resolution).

$f$ Ohno and Wendin [67].

Campbell and Papp's ones because, as discussed above, the latter were determined precisely from XPS data, those of Svensson included. In [7], level widths are also reported for the $O$-subshells. Values of $6 \mathrm{eV}, 5.7 \mathrm{eV}, 0.86 \mathrm{eV}$ and $1.33 \mathrm{eV}$ are given for the $5 p_{1 / 2}, 5 p_{3 / 2}, 5 d_{3 / 2}$ and $5 d_{5 / 2}$ levels of solid $\mathrm{Hg}$. As for the $N$-subshells, the values were not corrected for the instrumental resolution. Assuming for the $L_{1-3}$ level widths our values of $10.3(4) \mathrm{eV}$, $5.90(13) \mathrm{eV}$ and $6.46(12) \mathrm{eV}, \mathrm{O}_{2-5}$ widths of $9.0(8) \mathrm{eV}$, 8.1(7) eV, 1.6(4) eV and 1.1(2) eV are obtained from the widths of the transitions $L_{1}-O_{2,3}, L_{2}-O_{4}$ and $L_{3}-O_{5}$ presented in Table 3 . One sees that except for the $5 d_{5 / 2}$ level, Svensson's values are again 1-3 eV smaller than ours. These discrepancies between the level widths determined in the present work and those derived from XPS measurements are, however, not really surprising since it is well established that several factors complicate the experimental determination of natural lifetime broadenings in
XPS core-level spectroscopy. In particular, the asymmetry characterizing XPS peaks makes difficult the determination of the contribution of the lifetime broadening to the observed line shape [71]. This contribution is generally determined by considering the half-width at half-maximum (HWHM) on the low-binding energy side of the XPS peaks instead of the full-width at half-maximum (FWHM). However, lifetime broadenings extracted from HWHM widths are only correct if effects such as, e.g., the couplings of the core-electron transitions to many-electron excitations of the valence electrons, that may affect the positions and intensities of the profile centroids, are negligible.

Considering now the last column of Table 4, one sees that Krause and Oliver's semi-empirical values for the $K$-shell and $L_{1,2}$-subshells [53] are in agreement with present level widths. For the $L_{3}$-subshell, however, both values are consistent only if the interval of the combined error is extended to $2-\sigma$. Theoretical predictions 
from Chen et al. for the $K$-shell [55], $L_{2,3}$-subshells [56] and $M_{4,5}$-subshells [57] are well reproduced by our results. On the contrary, due to the above mentioned difficulty of theory to predict precise Coster-Kronig transition probabilities, the $M_{1}$ and to a lesser extent the $L_{1}$ and $M_{2,3}$ level widths obtained in our work are significantly overestimated by Chen et al. [40,56] calculations. A comparison with the theoretical level widths reported by McGuire [61-63] shows the same trend for theory to overestimate the experimental results but in this case the deviations are even more pronounced. However, for the $M_{4,5}$ subshells which are not affected by Coster-Kronig transitions, McGuire's predictions are consistent with present results. Data from non-relativistic MBT calculations by Ohno and Wendin [67] were also found for the $N$-subshells. In this study different approximations were probed. Results quoted in Table 4 correspond to the so-called A2 approximation corresponding to a frozen-core potential and relaxed Auger energy. As shown, the MBT results are smaller than McGuire's predictions and much closer to the experimental widths found in our work. For the $4 p_{3 / 2}$ level, however, a significantly smaller value than ours is provided by these MBT calculations. Finally, let us mention that a quick survey of the magnitude and trends of theoretical predictions for the total level widths can be obtained from the graphs published by Keski-Rahkonen and Krause [72]. However, as it is rather difficult to extract precise values from the logarithmic scales of these plots, we have renounced to quote these values in Table 4.

\section{Summary and concluding remarks}

High-resolution measurements of the fluorescence X-ray emission of liquid mercury were performed. X-ray transitions above $11 \mathrm{keV}$ were measured with a transmission DuMond-type crystal spectrometer, transitions below $11 \mathrm{keV}$ with a reflection von Hamos-type crystal spectrometer. From the observed spectra, precise and reliable data for the energies and widths of $K, L$ and $M$ X-ray transitions were determined. Energy shifts and/or line broadenings resulting from $M$ and $N$-satellites were accounted for in the data analysis. Solving by means of a least-squaresfit method the two linear systems of simultaneous equations built on the transition energies and transition widths obtained from the data analysis, the binding energies and natural widths of most core-levels of $\mathrm{Hg}$ could be deduced.

Bearden's transition energies [3] that are still used as references in many modern spectroscopy works were found to be inconsistent with present results for more than $30 \%$ of the measured transitions with deviations as big as $20 \mathrm{eV}$. In contrast to that a perfect agreement was found between our results and theoretical transition energies published recently [2].

For the binding energies of the ten core-levels that could be extracted from our work, a more or less satisfactory agreement was observed with Bearden and Burr's values [41] which, despite their age, still represent the main data base for binding energies of core-levels of mid- $Z$ and high- $Z$ elements. However, present binding energies were found to be systematically higher by about $1 \mathrm{eV}$, and for the $L_{1}$-subshell a discrepancy of $4 \mathrm{eV}$ was observed.

Present transition widths were compared to available experimental, semi-empirical and theoretical data. In general, existing information was found to be scarce and in most cases old. For the $K$ transitions the semi-empirical values from Krause and Oliver [53] are in agreement with our results, whereas the experimental values reported by Salem and Lee [54] lie significantly higher. For the widths of the few $L$ transitions quoted by Salem and Lee, smaller deviations are, however, observed. The comparison with results of theoretical calculations showed a satisfactory agreement except for the transitions involving $2 s$ or $3 s$ vacancy-states in the initial or final states.

The fifteen atomic level widths deduced from our measurements were compared principally to the recommended values reported recently by Campbell and Papp [5]. A fair agreement was found for most levels. The discrepancies observed for the $M_{2}$ and $N_{2,3}$-subshells were discussed and tentative explanations were proposed. Results of theoretical predictions from different sources were also considered. As for the transition widths, it was found that theory provides reliable predictions, except for the levels whose lifetime broadenings are dominated by Coster-Kronig transitions. In these cases, the calculations, except the MBT ones, overestimate considerably the experimental level widths.

We would like to acknowledge the assistance of J.P. Santos, J.P. Marques and F. Parente (Lisboa), P. Indelicato (Paris) and J.P. Desclaux (Grenoble) for the computation of the $L_{2,3}$ Coster-Kronig yields of $\mathrm{Hg}$. The latter were very useful for the determination of the theoretical line shapes of the $M$ and $N$-shell satellites employed in the data analysis. We also express our thanks to M. Polasik (Torun) for providing the MCDF calculations concerning the binding energies of the $\mathrm{Hg}$ core-levels. This work was partly supported by the Swiss National Science Foundation.

\section{References}

1. T. Papp, J.L. Campbell, J.A. Maxwell, J.-X. Wang, W.J. Teesdale, Phys. Rev. A 45, 1711 (1992)

2. R.D. Deslattes et al., Rev. Mod. Phys. 75, 35 (2003)

3. J.A. Bearden, Rev. Mod. Phys. 39, 78 (1967)

4. J.C. Fuggle, N. Martensson, J. Electron Spectros. Relat. Phenomena 21, 275 (1980)

5. J.L. Campbell, T. Papp, At. Data Nucl. Data Tables 77, $1(2001)$

6. O. Keski-Rahkonen, G. Materlik, B. Sonntag, J. Tulkki, J. Phys. B: At. Mol. Phys. 17, L121 (1984)

7. S. Svensson et al., J. Electron Spectros. Relat. Phenomena 9, 51 (1976)

8. R. Nyholm, N. Martensson, Phys. Rev. B 36, 20 (1987)

9. A. Mühleisen, M. Budnar, J.-Cl. Dousse, Phys. Rev. A 54, 3852 (1996)

10. J.-Cl. Dousse, J. Hoszowska, Phys. Rev. A 56, 4517 (1997)

11. J. Hoszowska, J.-Cl. Dousse, Ch. Rhême, Phys. Rev. A 50, $123(1994)$ 
12. W. Beer, P.F.A. Goudsmit, L. Knecht, Nucl. Instrum. Meth. A 219, 322 (1984)

13. O.I. Sumbaev, Soviet Phys. JETP 5, 1042 (1957)

14. W. Schwitz, Nucl. Instrum. Meth. 154, 95 (1978)

15. R.C. Sharma et al., Nucl. Instrum. Meth. 130, 305 (1975)

16. B. Galley, J.-Cl. Dousse, Phys. Rev. A 50, 3058 (1994)

17. Ch. Herren, J.-Cl. Dousse, Phys. Rev. A 53, 717 (1996)

18. Ch. Herren, J.-Cl. Dousse, Phys. Rev. A 56, 2750 (1997)

19. R.D. Deslattes, E.G. Kessler, Jr, in Atomic Inner-Shell Physics, edited by B. Crasemann (Plenum, New York, 1985), pp. 181-235

20. R.D. Deslattes, M. Tanaka, G.L. Greene, A. Henins, E.G. Kessler, Jr, IEEE Trans. Instrum. Meas. 36, 166 (1987)

21. J. Hoszowska, J.-Cl. Dousse, J. Kern, Ch. Rhême, Nucl. Instrum. Meth. A 376, 129 (1996)

22. J. Szlachetko, J.-Cl. Dousse, J. Hoszowska, M. Berset, W. Cao, M. Szlachetko, M. Kavcic, Rev. Sci. Instrum. 78, 093102 (2007)

23. P.J. Mohr, B.N. Taylor, D.B. Newell, J. Phys. Chem. Ref. Data 37, 1187 (2008)

24. F. James, M. Roos, Comput. Phys. Commun. 10, 343 (1975)

25. G.K. Wertheim, M.A. Butler, K.W. West, D.N.E. Buchanan, Rev. Sci. Instrum. 45, 11 (1974)

26. J.H. Scofield, At. Data Nucl. Data Tables 14, 121 (1974)

27. T.A. Carlson, C.W. Nestor, Phys. Rev. A 8, 2887 (1973)

28. T. Mukoyama, Y. Ito, Nucl. Instrum. Meth. B 87, 26 (1994), and references therein

29. F. Parente, M.H. Chen, B. Crasemann, H. Mark, At. Data Nucl. Data Tables 26, 383 (1981)

30. J.H. Scofield, in Atomic Inner-Shell Processes, edited by B. Crasemann (Academic Press, New York, 1975), Vol. I, p. 265

31. Z. Rudzikas, in Theoretical Atomic Spectroscopy, Cambridge Monographs on Atomic, Molecular and Chemical Physics, Vol. 7 (Cambridge University Press, 1997)

32. J.P. Santos, J.P. Marques, F. Parente, P. Indelicato, J.P. Desclaux, At. Data Nucl. Data Tables 76, 49 (2000)

33. J.P. Santos, private communication

34. M.O. Krause, J. Phys. Chem. Ref. Data 8, 307 (1979)

35. E.J. McGuire, Phys. Rev. A 5, 1052 (1972)

36. W. Uchai, C.W. Nestor, Jr, S. Raman, C.R. Vane, At. Data Nucl. Data Tables 34, 201 (1986)

37. J. Härtwig, G. Holzer, J. Wolf, E. Förster, J. Appl. Crystallogr. 26, 539 (1994)

38. P.-A. Raboud, M. Berset, J.-Cl. Dousse, Y.-P. Maillard, Phys. Rev. A 65, 022512 (2002)

39. P. Indelicato, S. Boucard, E. Lindroth, Eur. Phys. J. D 3, 29 (1998)

40. M.H. Chen, B. Crasemann, H. Mark, Phys. Rev. A 27, $2989(1983)$
41. J.A. Bearden, A.F. Burr, Rev. Mod. Phys. 39, 125 (1967)

42. E. Storm, H.I. Israel, Nucl. Data Tables 7, 565 (1970)

43. M. Polasik, private communication

44. K.-N. Huang et al., At. Data Nucl. Data Tables 18, 233 (1976)

45. M.H. Chen, B. Crasemann, M. Aoyagi, K. Huang, H. Mark, At. Data Nucl. Data Tables 26, 561 (1981)

46. P.-A. Raboud, J.-Cl. Dousse, J. Hoszowska, I. Savoy, Phys. Rev. A 61, 12507 (2000)

47. G.P. Williams, http://xray.uu.se/hypertext/EBindEnergies.html

48. M. Polasik, Phys. Rev. A 52, 227 (1995), and references therein

49. I.P. Grant, B.J. McKenzie, P.H. Norrington, D.F. Mayers, N.C. Pyper, Comput. Phys. Commun. 21, 207 (1980)

50. G.C. Nelson, W. John, B.G. Saunders, Phys. Rev. 187, 1 (1969)

51. G.C. Nelson, W. John, B.G. Saunders, Phys. Rev. A 2, $542(1970)$

52. G.C. Nelson, B.G. Saunders, J. Phys. Colloques 32, C4-97 (1971)

53. M.O. Krause, J.H. Oliver, J. Phys. Chem. Ref. Data 8, 329 (1979)

54. S.I. Salem, P.L. Lee, At. Data Nucl. Data Tables 18, 233 (1976)

55. M.H. Chen, B. Crasemann, H. Mark, Phys. Rev. A 21, 436 (1980)

56. M.H. Chen, B. Crasemann, H. Mark, Phys. Rev. A 24, 177 (1981)

57. M.H. Chen, B. Crasemann, H. Mark, Phys. Rev. A 21, 449 (1980)

58. E.G. Kessler, Jr, R.D. Deslattes, D. Girard, W. Schwitz, L. Jacobs, O. Renner, Phys. Rev. A 26, 2696 (1982)

59. J.N. Cooper, Phys. Rev. 61, 234 (1941)

60. J.H. Williams, Phys. Rev. 45, 71 (1934)

61. E.J. McGuire, Phys. Rev. A 5, 1043 (1972)

62. E.J. McGuire, Phys. Rev. A 9, 1840 (1974)

63. E.J. McGuire, Phys. Rev. A 13, 1288 (1976)

64. O. Mauron, J.-Cl. Dousse, S. Baechler, M. Berset, Y.-P. Maillard, P.-A. Raboud, J. Hoszowska, Phys. Rev. A 67 032506 (2003)

65. E.J. McGuire, Phys. Rev. A 3, 587 (1971)

66. M.H. Chen, B. Crasemann, H. Mark, At. Data Nucl. Data Tables 24, 13 (1979)

67. M. Ohno, G. Wendin, Phys. Rev. A 31, 2318 (1985)

68. M. Ohno, Phys. Rev. B 29, 3127 (1984)

69. I. Salem, P.L. Lee, Phys. Rev. A 10, 2033 (1974)

70. K.J. Rawlings, B.J. Hopkins, S.D. Foulias, J. Electron Spectrosc. 18, 213 (1980)

71. J.C. Fuggle, S.F. Alvarado, Phys. Rev. A 22, 1615 (1980)

72. O. Keski-Rahkonen, M. Krause, At. Data Nucl. Data Tables 14, 139 (1974) 